\title{
Efficient sensitivity analysis method for chaotic dynamical systems
}

\author{
Haitao Liao \\ Address: Chinese Aeronautical Establishment, Beijing 100012, China \\ Phone:086-10-15810533731 Email: ht0819@163.com or liaoht@cae.ac.cn
}

\begin{abstract}
:
The direct differentiation and improved least squares shadowing methods are both developed for accurately and efficiently calculating the sensitivity coefficients of time averaged quantities for chaotic dynamical systems. The key idea is to recast the time averaged integration term in the form of differential equation before applying the sensitivity analysis method. An additional constraint-based equation which forms the augmented equations of motion is proposed to calculate the time averaged integration variable and the sensitivity coefficients are obtained as a result of solving the augmented differential equations. The application of the least squares shadowing formulation to the augmented equations results in an explicit expression for the sensitivity coefficient which is dependent on the final state of the Lagrange multipliers. The LU factorization technique to calculate the Lagrange multipliers leads to a better performance for the convergence problem and the computational expense. Numerical experiments on a set of problems selected from the literature are presented to illustrate the developed methods. The numerical results demonstrate the correctness and effectiveness of the present approaches and some short impulsive sensitivity coefficients are observed by using the direct differentiation sensitivity analysis method.
\end{abstract}

KeyWords: Sensitivity; Time averaged variable; Direct differentiation method; Improved least squares shadowing method;

\section{Introduction}

Sensitivity analysis is used to evaluate the rate of change of performance measure with respect to system parameter changes. The knowledge of sensitivity for dynamical systems is of considerable interest in structural dynamic reduction[1], optimal control[2], reliability analysis[3], and uncertainty analysis[4]. The sensitivity analysis can be carried out by using different methods such as the finite difference method, the direct differentiation method, and the adjoint variable method[5][6]. The sensitivity of performance measure computed using the direct and adjoint methods is essentially identical. Although the finite difference method is easy to implement but it suffers from computational inefficiency and possible errors. On the contrary, the adjoint variables can be successively or repeatedly used for the evaluation of the sensitivity for different optimization variables. More information on the subject and the sensitivity theory can be found in the several classical books [7][8][9].

Many studies have been devoted to investigate the sensitivities of periodic solutions for nonlinear systems[10]. For instance, Liao[11] studied the sensitivity and robust stability of periodic motions for fractional order nonlinear dynamic systems by means of constrained 
optimization harmonic balance method[12]. Since time delay has an important effect on the performance of dynamic systems, research activities on periodic motions and dynamics of time delayed system have been carried out in [13] and the sensitivities of the Duffing oscillator subjected to various kinds of time delays are analyzed. In [14], the sensitivity analysis is extended to fractional-order nonlinear system with delay as nonlinear fractional delay differential equations are a very recent topic. Recently, the sensitivities of an airfoil system with various nonlinear structures are studied in [15].

Studying dynamical behavior of chaotic systems has attracted considerable attention in recent years. It is well known that sensitive dependence characterizes the unpredictability of chaotic phenomenon and trajectories adjacent to each other in chaotic systems become divergent in finite time[16]. Due to the extreme initial condition sensitivity, conventional approaches of sensitivity analysis are inadequate for solving the sensitivity problem of chaotic dynamical systems and this category sensitivity problem becomes an open question[17][18].

Mathematically, the sensitivity analysis problem of chaotic dynamical system is known to be ill-posed[19]. Ill-posed problems are inherently unstable and very sensitive to the inaccuracy in input data. Therefore, development of new computational schemes is necessary to overcome the numerical ill-posed problem.

In scientific and engineering community, considerable attention has been paid to the study of the long time statistical averaged quantities. Until now, relatively little work has been done addressing sensitivity computation of statistical quantities in chaotic dynamical systems since the distance between neighboring trajectories grows exponentially with time. For example, Wang[20] originally presented the Lyapunov eigenvector decomposition method to obtain the dynamic response sensitivity of chaotic dynamical systems. Although the Lyapunov eigenvector decomposition method has been found to be successful to predict the sensitivity coefficients of chaotic dynamical systems, but it has some potential limitations. The cost associated with this method is proportional to the number of Lyapunov exponents multiplied by the system dimension. The increase in the system dimension can result in a significant increase in the computational expense.

To alleviate the computational cost, a method named the Least Squares Shadowing(LSS) method has been developed in [21] to predict the sensitivity coefficients of chaotic limit cycle systems. Motivated by the pseudo-orbit shadowing theory in dynamical systems, the sensitivity analysis is transformed into a minimization problem for a least-squares objective function.

As an essential part of stability and ergodic theory, the shadowing property (also called the pseudo orbit tracing property) means that pseudo orbits generated by integration of the system equations are shaded by true orbits of the original system and therefore the numerically detected behavior of the system indeed reflects its real dynamical behavior. The main aim of the shadowing property is to obtain shadowing of approximate trajectories in a given dynamical system by true orbits of the system while the shadowing lemma establishes the existence of a true trajectory that remains close to a given pseudo trajectory. In addition, it is worth noting here that the ergodic hypothesis in chaotic systems should be satisfied when the shadowing theory is applied. For more clear and complete knowledge about the shadowing theory can be found in the monographs [22][23]. 
Based on the shadowing Lemma, traditional mathematical shadowing theory is devised for hyperbolic systems, which is characterized by the presence of expanding and contracting directions for derivatives. However, most physical systems (e.g. Earth's atmosphere) are nonhyperbolic. Although there is evidence that non hyperbolic systems also have the shadowing property, however, it is very difficult to prove that the shadowing theory can be applied to the non hyperbolic systems. Thus, finding the shadowing trajectories for non-hyperbolic systems has been the desire for many researchers.

Following the work of Wang[21], the convergence of the LSS method has been studied by Wang[24]. In a subsequent paper, convergence conditions are presented for the LSS method in [25]. However, the need for solving a large linear system of the LSS approach places a restriction on the hardware resources as large amounts of memory are required.

The multigrid-in-time technique for the LSS method has been investigated in [26] and several multigrid-in-time schemes including classic geometric multigrid, matrix restriction multigrid and solution restriction multigrid are considered. However, the computational cost and memory requirements due to a large number of time steps are still prohibitively large.

The checkpointing techniques which store only these solutions states corresponding to a small number of specific time steps known as check points are employed in [27]. However, there is no universal rule to select check points. Therefore, there is a need to develop the efficient algorithms to reduce the computational cost and memory requirements for the LSS scheme and subsequently improve the overall computational effort.

The motivation of the present paper is to develop the sensitivity computation methods for nonlinear dynamical systems. The developed approaches are aimed at calculation of sensitivity coefficients associated with time averaged quantity. The time averaged integral term is converted into differential equation and the augmented differential equations are then constructed to predict the sensitivity coefficients.

The remaining sections of the article are laid out as follows: section 2 outlines the augmented equations of motion behind the sensitivity analysis problem and the direct differentiation formulation used to compute the sensitivity coefficients is provided in section 3. In section 4, the modified least squares shadowing method based on the augmentation equations of motion is derived and the algorithm used to implement the procedure computationally is given in section 5 . Numerical results along with some discussion and analysis are presented in section 6 to verify the developed method. Finally, the paper ends with a summary highlighting the most significant contributions in section 7.

\section{Augmentation of the original governing equation}

In order to perform sensitivity analysis, the time averaged variable is defined and the augmented system of differentiation equations is formed.

Consider the following differential equation for sensitivity analysis:

$$
\frac{d \boldsymbol{u}(t)}{d t}=\boldsymbol{f}(\boldsymbol{u}(t), p)
$$


where $\boldsymbol{u}(t)$ is a $n$-dimensional state vector with initial condition $\left.\boldsymbol{u}(t)\right|_{t=0}=\boldsymbol{u}_{0}$ and $\boldsymbol{f}$ is a nonlinear $n$-dimensional vector valued function that depends on a control parameter $p$.

In dynamical systems, the long-time behavior of statistical averaged quantities is of major importance in numerous applications in the realm of aeronautic and atmospheric fields. Therefore, the time averaged quantity related to $J(\boldsymbol{u}(t), p)$ is usually considered as the sensitivity related function for the sensitivity analysis problem. On the other hand, $J(\boldsymbol{u}(t), p)$ can be viewed as a function of time $t$. By virtue of the integral of $J(\boldsymbol{u}(t), p)$ up to time point $t$, the time-averaged variable $e(t)$ over the time interval from 0 to $t$ is defined as:

$$
e(t)=\frac{1}{t} \int_{0}^{t} J\left(\boldsymbol{u}\left(t^{\prime}\right), p\right) d t^{\prime}
$$

Upon differentiating Eq.(2) with respect to $t$, the following expression is obtained:

$$
\frac{d e(t)}{d t}=g(\boldsymbol{u}(t), e(t), p)=\frac{J(\boldsymbol{u}(t), p)-e(t)}{t}
$$

Taking the time derivative of the expression in Eq.(2) results in a differential equation which governs the time evolution of time averaged variable $e(t)$. The objective is to compute sensitivities associated with $e(t)$. To achieve this goal, the auxiliary equation in Eq.(3) should be considered and a set of augmented differential equations is formed:

$$
\frac{d \boldsymbol{U}(t)}{d t}=\boldsymbol{h}(\boldsymbol{U}(t), p)=\left[\begin{array}{c}
f(\boldsymbol{u}(t), p) \\
g(\boldsymbol{u}(t), e(t), p)
\end{array}\right]
$$

where the augmented state and initial conditions are defined as

$$
\boldsymbol{U}(t)=\left[\begin{array}{l}
\boldsymbol{u}(t) \\
e(t)
\end{array}\right], \boldsymbol{U}\left(t_{0}\right)=\left[\begin{array}{l}
\boldsymbol{u}\left(t_{0}\right) \\
e\left(t_{0}\right)
\end{array}\right]
$$

The expression in Eq.(4) augments the system in Eq.(1) so the time dependent variable $e(t)$ can be determined. The augmented state differential equations lay the foundation for sensitivity analysis problem. In addition, it should be noted that the original differential system in Eq.(1) can be augmented with many time averaged variables.

\section{The direct differentiation sensitivity formulation for the augmented system}

The direct differentiation method involves deriving analytical expressions for the response function by directly differentiating Eq.(4) with respect to the influence parameter $p$ : 


$$
\frac{d \boldsymbol{V}_{d}(t)}{d t}=\frac{\partial \boldsymbol{h}(\boldsymbol{U}(t), p)}{\partial p}+\frac{\partial \boldsymbol{h}(\boldsymbol{U}(t), p)}{\partial \boldsymbol{U}} \boldsymbol{V}_{d}(t)
$$

where the first term in this expression represents an explicit dependence on variable $p$ and the second term shows the implicit dependence through dynamic response $\boldsymbol{U}$. The calculation of $\frac{\partial \boldsymbol{h}(\boldsymbol{U}(t), p)}{\partial p}$ and $\frac{\partial \boldsymbol{h}(\boldsymbol{U}(t), p)}{\partial \boldsymbol{U}}$ is generally straight forward. On the contrary, the evaluation of $\frac{\partial e(t)}{\partial p}$ is transformed into the calculation the final element of $\boldsymbol{V}_{d}(t)=\frac{\partial \boldsymbol{U}(t)}{\partial p}$ according to Eq.(6).

The key of computing the system sensitivity is to compute the derivatives of the state variable $e(t)$ with respect to the system's parameters. Eq.(3) is used as an extra equation for sensitivity analysis.

Based on the augmentation equations in Eq.(4), the sensitivity function is estimated by using the implicit function theory. The advantage of the new formulation is that it provides the exact derivative for the time averaged variable with respect to the influence parameter.

It is worth to mention that the proposed direct differentiation method belongs to the conventional sensitivity analysis method. The application of the conventional sensitivity analysis method to analyze the sensitivity of chaotic systems is reported in sections 2 and 3 of Wang[21] and the limitations of this method are also described. More discussion about the conventional sensitivity analysis method can be referred to section 3 of Wang[21].

\section{The improved least squares shadowing formulation for the augmented system}

Based on the shadowing theory, the improved least squares shadowing method is derived in this section to solve the ill-posed sensitivity problem of chaotic systems.

As shown in Wang[21] and section 6, the direct sensitivity analysis method may not be efficient for solving some problems. Therefore, an alternative approach such as the LSS method is a better choice. In the following, the least squares shadowing method shown in Fig.1 (borrowed from [26]) is applied to the governing equations in Eq.(4). 

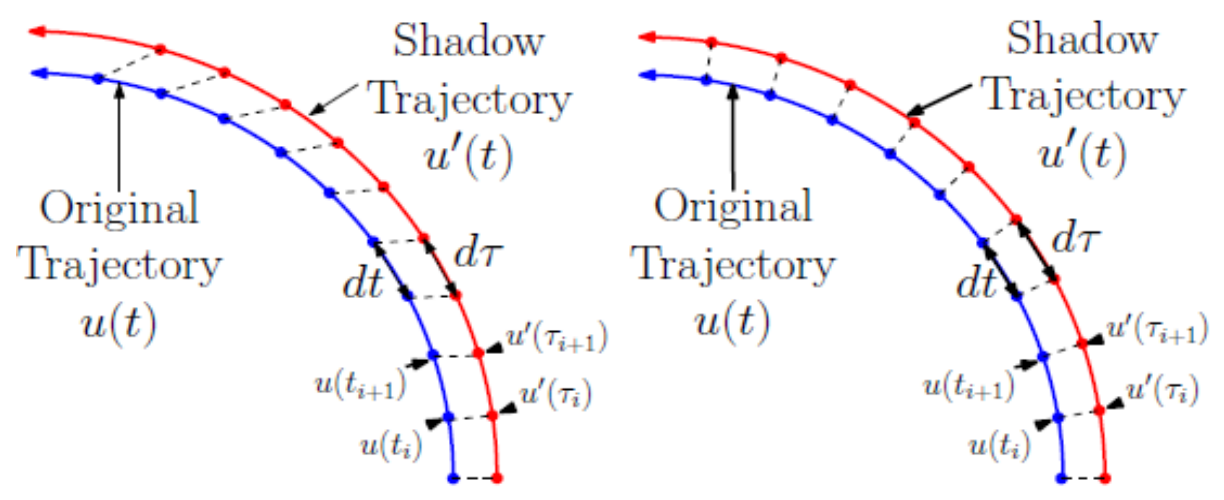

Fig.1 The shadow trajectory principle for sensitivity analysis

Making use of the shadowing lemma[23][26], the sensitivity problem can be translated into the least squares shadowing problem:

$$
\begin{array}{cc}
\text { minimize } & \frac{1}{2} \int_{0}^{T}\left(\left\|\boldsymbol{U}(\tau(t))-\boldsymbol{U}_{r}(t)\right\|^{2}+\alpha^{2}\left(\frac{d(\tau(t))}{d t}-1\right)^{2}\right) d t \\
\text { s.t } & \frac{d \boldsymbol{U}(\tau(t))}{d t}=\boldsymbol{h}(\boldsymbol{U}(\tau(t)), p)
\end{array}
$$

where $\boldsymbol{U}_{r}(t)$ denotes the reference solution at $p$ and $\tau(t)$ represents the time transformation for solving Eq.(7). $\alpha$ means the weighting constant parameter.

Let $\boldsymbol{U}_{l s s}(t, p)$ and $\tau_{l s s}(t, p)$ be the solutions for the least squares constrained optimization problem in Eq.(7). In order to analyze the sensitivity, the following variables are introduced:

$$
\begin{aligned}
& \boldsymbol{V}(t)=\frac{d\left(\boldsymbol{U}_{l s s}\left(\tau_{l s s}(t, p), p\right)-\boldsymbol{U}_{r}(t)\right)}{d p}, \\
& \eta(t)=\frac{d}{d p}\left(\frac{d \tau_{l s s}(t, p)}{d t}-1\right)
\end{aligned}
$$

For infinitesimal $\delta p, \tau_{l s s}(t, p+\delta p)$ and $\boldsymbol{U}_{l s s}\left(\tau_{l s s}(t, p+\delta p), p+\delta p\right)$ can be derived according to Eq.(8) as follows:

$$
\begin{aligned}
& \tau_{l s s}(t, p+\delta p)=\int_{0}^{t}\left(1+\eta\left(t^{\prime}\right) \delta p\right) d t^{\prime} \\
& \boldsymbol{U}_{l s s}\left(\tau_{l s s}(t, p+\delta p), p+\delta p\right)=\boldsymbol{U}_{r}(t)+\boldsymbol{V}(t) \delta p
\end{aligned}
$$

With the help of the Taylor expansion technique, differentiating $\boldsymbol{U}_{l s s}\left(\tau_{l s s}(t, p+\delta p), p+\delta p\right)$ in Eq.(9) with respect to the time variable yields: 


$$
\begin{aligned}
& \frac{d\left(\boldsymbol{U}_{r}(t)+\boldsymbol{V}(t) \delta p\right)}{d t}=\frac{d \boldsymbol{U}_{r}(t)}{d t}+\frac{d \boldsymbol{V}(t)}{d t} \delta p=\frac{d \boldsymbol{U}_{l s s}\left(\tau_{l s s}(t, p+\delta p), p+\delta p\right)}{d t} \\
& =\left.\frac{d \boldsymbol{U}_{l s s}(\tau, p+\delta p)}{d \tau}\right|_{\tau=\tau_{l s s}(t, p+\delta p)} \frac{d \tau_{l s s}(t, p+\delta p)}{d t} \\
& =\boldsymbol{h}\left(\boldsymbol{U}_{l s s}\left(\tau_{l s s}(t, p+\delta p), p+\delta p\right), p+\delta p\right)(1+\eta(t) \delta p) \\
& =\left(\boldsymbol{h}\left(\boldsymbol{U}_{r}(t), p\right)+\frac{\partial \boldsymbol{h}\left(\boldsymbol{U}_{r}(t), p\right)}{\partial \boldsymbol{U}} \boldsymbol{V}(t) \delta p+\frac{\partial \boldsymbol{h}\left(\boldsymbol{U}_{r}(t), p\right)}{\partial p} \delta p\right)(1+\eta(t) \delta p) \\
& =\boldsymbol{h}\left(\boldsymbol{U}_{r}(t), p\right)+\left(\frac{\partial \boldsymbol{h}\left(\boldsymbol{U}_{r}(t), p\right)}{\partial \boldsymbol{U}} \boldsymbol{V}(t)+\frac{\partial \boldsymbol{h}\left(\boldsymbol{U}_{r}(t), p\right)}{\partial p}+\eta(t) \boldsymbol{h}\left(\boldsymbol{U}_{r}(t), p\right)\right) \delta p+o\left((\delta p)^{2}\right)
\end{aligned}
$$

Utilizing the relationship $\frac{d \boldsymbol{U}_{r}(t)}{d t}=\boldsymbol{h}\left(\boldsymbol{U}_{r}(t), p\right)$ and equating the same term of $\delta s$ in Eq.(10) get

$$
\frac{d \boldsymbol{V}(t)}{d t}=\frac{\partial \boldsymbol{h}\left(\boldsymbol{U}_{r}(t), p\right)}{\partial \boldsymbol{U}} \boldsymbol{V}(t)+\frac{\partial \boldsymbol{h}\left(\boldsymbol{U}_{r}(t), p\right)}{\partial p}+\eta(t) \boldsymbol{h}\left(\boldsymbol{U}_{r}(t), p\right)
$$

With the aid of Eqs.(8), (9) and (11), the nonlinear least squares shadowing problem in Eq.(7) can be replaced by the following constrained optimization problem

$$
\begin{aligned}
& \text { minimize } \frac{1}{2} \int_{0}^{T}\left(\boldsymbol{V}(t)^{T_{r}} \boldsymbol{V}(t)+\alpha^{2}(\eta(t))^{2}\right) d t \\
& \text { s.t } \quad \frac{d \boldsymbol{V}(t)}{d t}=\frac{\partial \boldsymbol{h}\left(\boldsymbol{U}_{r}(t), p\right)}{\partial \boldsymbol{U}} \boldsymbol{V}(t)+\frac{\partial \boldsymbol{h}\left(\boldsymbol{U}_{r}(t), p\right)}{\partial p}+\eta(t) \boldsymbol{h}\left(\boldsymbol{U}_{r}(t), p\right)
\end{aligned}
$$

where $T_{r}$ denotes the mathematical transpose symbol.

Combining the objective function and the constraints in a single minimization problem yields

$$
L=\int_{0}^{T}\left[\frac{\left(\boldsymbol{V}(t)^{T_{r}} \boldsymbol{V}(t)+\alpha^{2}(\eta(t))^{2}\right)}{2}+\mathbf{W}^{T_{r}}\left(\frac{\partial \boldsymbol{h}\left(\boldsymbol{U}_{r}(t), p\right)}{\partial \boldsymbol{U}} \boldsymbol{V}(t)+\frac{\partial \boldsymbol{h}\left(\boldsymbol{U}_{r}(t), p\right)}{\partial p}+\eta(t) \boldsymbol{h}\left(\boldsymbol{U}_{r}(t), p\right)-\frac{d \boldsymbol{V}(t)}{d t}\right)\right] d t
$$

where $L$ is the Lagrange function and $\mathbf{W}$ is a vector of Lagrange multipliers.

The first-order optimality conditions or Karush-Kuhn-Tucker(KKT) conditions for the minimization of $L$ are as follows: 


$$
\begin{aligned}
& \frac{d \boldsymbol{V}(t)}{d t}=\frac{\partial \boldsymbol{h}\left(\boldsymbol{U}_{r}(t), p\right)}{\partial \boldsymbol{U}} \boldsymbol{V}(t)+\frac{\partial \boldsymbol{h}\left(\boldsymbol{U}_{r}(t), p\right)}{\partial p}+\eta(t) \boldsymbol{h}\left(\boldsymbol{U}_{r}(t), p\right) \\
& \alpha^{2} \eta(t)=-\boldsymbol{W}(t)^{T_{r}} \boldsymbol{h}\left(\boldsymbol{U}_{r}(t), p\right) \\
& \frac{d \boldsymbol{W}(t)}{d t}=-\left(\frac{\partial \boldsymbol{h}\left(\boldsymbol{U}_{r}(t), p\right)}{\partial \boldsymbol{U}}\right)^{T_{r}} \boldsymbol{W}(t)-\boldsymbol{V}(t), \boldsymbol{W}(0)=\boldsymbol{W}(T)=0
\end{aligned}
$$

The derivative of the system response $\left.e(t)\right|_{t=T}$ with respect to the system parameter $p$ is governed by the final element of $\left.\boldsymbol{V}(t)\right|_{t=T}$. Once the Lagrange multipliers are calculated, the sensitivity coefficients $\left.\boldsymbol{V}(t)\right|_{t=T}$ are obtained according to Eq.(14).

\section{Numerical calculation for the improved least squares shadowing sensitivity problem}

In this section, the finite difference method with trapezoidal discretization scheme coupled with LU factorization technique are adopted to solve the improved least squares shadowing sensitivity problem in section 4 .

\subsection{Discretization for the KKT system}

Based on the literature[21][26], Eq.(14) can be discreted following two different approaches: direct method and adjoint method. Both methods result in the same linear equations and boundary conditions. In the following, discretion set of linear equations for the system considered in Eq.(14) is derived and solved.

Eq.(14) is discreted in physical time using finite difference method and the trapezoidal rule. The time interval $\left[t_{0}, T+t_{0}\right]$ is equally divided into a set of $m$ subintervals. The time step is denoted by $\Delta t=T / \mathrm{m}$. The corresponding sampled time points are $t_{i}=t_{0}+i \Delta t$ where $i=1,2, \ldots, m$ and $m$ is a sufficiently large positive integer.

Using the above discretization scheme, a set of linear equations in terms of $\overline{\boldsymbol{V}}=\left[\boldsymbol{V}_{0}^{T}, \boldsymbol{V}_{1}^{T}, \boldsymbol{V}_{2}^{T}, \mathrm{~L}, \boldsymbol{V}_{m}^{T}\right]^{T_{r}} \quad, \quad \overline{\boldsymbol{\eta}}=\left[\eta_{1}, \eta_{2}, \mathrm{~L}, \eta_{m}\right]^{T_{r}} \quad$ and $\quad \overline{\boldsymbol{W}}=\left[\boldsymbol{W}_{1}^{T}, \boldsymbol{W}_{2}^{T}, \mathrm{~L}, \boldsymbol{W}_{m}^{T}\right]^{T_{r}}$ where $\boldsymbol{V}_{i}=\left.\boldsymbol{V}(t)\right|_{t=t_{i}}, \eta_{i}=\left.\eta(t)\right|_{t=t_{i}}$, and $\boldsymbol{W}_{i}=\left.\boldsymbol{W}(t)\right|_{t=t_{i}}$, can be constructed to solve the KKT system in Eq.(14):

$$
\left[\begin{array}{ccc}
\boldsymbol{I} & 0 & \boldsymbol{B}^{T_{r}} \\
0 & \alpha^{2} \boldsymbol{I} & \boldsymbol{C}^{T_{r}} \\
\boldsymbol{B} & \boldsymbol{C} &
\end{array}\right]\left(\begin{array}{c}
\overline{\boldsymbol{V}} \\
\overline{\boldsymbol{\eta}} \\
\overline{\boldsymbol{W}}
\end{array}\right)=\left(\begin{array}{c}
0 \\
0 \\
-\overline{\boldsymbol{b}}
\end{array}\right)
$$

where $\boldsymbol{I}$ represents the identity matrix and matrices $\boldsymbol{B}$ and $\mathbf{C}$ are defined as follows 


$$
\begin{gathered}
\boldsymbol{B}=\left[\begin{array}{ccccc}
\boldsymbol{F}_{0} & \boldsymbol{G}_{1} & & & \\
& \boldsymbol{F}_{1} & \boldsymbol{G}_{2} & & \\
& & \mathrm{O} & \mathrm{O} & \\
& & & \boldsymbol{F}_{m-1} & \boldsymbol{G}_{m}
\end{array}\right], \boldsymbol{B}^{T_{r}}=\left[\begin{array}{cccc}
\boldsymbol{F}_{0}^{T_{r}} & & & \\
\boldsymbol{G}_{1}^{T} & \boldsymbol{F}_{1}^{T_{r}} & & \\
& \boldsymbol{G}_{2}^{T} & \mathrm{O} & \\
& & \mathrm{O} & \boldsymbol{F}_{m-1}^{T_{r}} \\
& & & \boldsymbol{G}_{m}^{T}
\end{array}\right] \\
\mathbf{C}=\operatorname{diag}\left(\begin{array}{llll}
\hat{\boldsymbol{h}}_{1} & , \hat{\boldsymbol{h}}_{2} & , \mathrm{~L} & , \hat{\boldsymbol{h}}_{m}
\end{array}\right)
\end{gathered}
$$

with

$$
\begin{aligned}
& \boldsymbol{F}_{i}=\frac{\boldsymbol{I}}{\Delta t}+\frac{1}{2} \frac{\partial \boldsymbol{h}\left(\boldsymbol{U}_{i}(t), p\right)}{\partial \boldsymbol{U}}, \boldsymbol{G}_{i}=-\frac{\boldsymbol{I}}{\Delta t}+\frac{1}{2} \frac{\partial \boldsymbol{h}\left(\boldsymbol{U}_{i}(t), p\right)}{\partial \boldsymbol{U}} \\
& \boldsymbol{b}_{i}=\frac{1}{2}\left[\frac{\partial \boldsymbol{h}\left(\boldsymbol{U}_{i-1}(t), p\right)}{\partial p}+\frac{\partial \boldsymbol{h}\left(\boldsymbol{U}_{i}(t), p\right)}{\partial p}\right], \hat{\boldsymbol{h}}_{i}=\frac{1}{2}\left[\boldsymbol{h}\left(\boldsymbol{U}_{i-1}(t), p\right)+\boldsymbol{h}\left(\boldsymbol{U}_{i}(t), p\right)\right]
\end{aligned}
$$

According to the first row of Eq.(15), the sensitivity coefficients of $\boldsymbol{V}_{m}$ evaluated at the end of time $T$ is calculated as follows:

$$
\boldsymbol{V}_{m}=-\boldsymbol{G}_{m}^{T_{r}} \cdot \boldsymbol{W}_{m}
$$

It can be seen from Eq.(18) that only the final state of the Lagrange multipliers in Eq.(15) should be calculated and all sensitivity coefficients can be calculated directly using $\boldsymbol{G}_{m}$ and $\boldsymbol{W}_{m}$ without the need for solving additional solutions $\boldsymbol{V}_{i}\left(i=0,1,2, \ldots m_{-}\right)$and $\overline{\boldsymbol{\eta}}$.

On the contrary, the original LSS method requires to calculate $\overline{\boldsymbol{V}}, \overline{\boldsymbol{\eta}}$ and $\overline{\boldsymbol{W}}$ at first in order to further analyze the sensitivity coefficients of some given objective function.

Eliminating $\overline{\boldsymbol{V}}, \overline{\boldsymbol{\eta}}$ and regrouping the equations yield the following linear matrix equation:

$$
\left(\boldsymbol{B} B^{T_{r}}+\frac{1}{\alpha^{2}} \boldsymbol{C} \boldsymbol{C}^{T_{r}}\right) \overline{\boldsymbol{W}}=\boldsymbol{A} \overline{\boldsymbol{W}}=\overline{\boldsymbol{b}}
$$

where the block tridiagonal matrix $\boldsymbol{A}$ is defined as

$$
\boldsymbol{A}=\left[\begin{array}{ccccc}
\boldsymbol{S}_{1} & \boldsymbol{R}_{1}^{T_{r}} & & & \\
\boldsymbol{R}_{1} & \boldsymbol{S}_{2} & \boldsymbol{R}_{2}^{T_{r}} & & \\
& \boldsymbol{R}_{2} & \mathrm{O} & \mathrm{O} & \\
& & \mathrm{O} & \boldsymbol{S}_{m-1} & \boldsymbol{R}_{m-1}^{T_{r}} \\
& & & \boldsymbol{R}_{m-1} & \boldsymbol{S}_{m}
\end{array}\right]
$$


with $\boldsymbol{S}_{i}=\boldsymbol{F}_{i-1} \boldsymbol{F}_{i-1}^{T_{r}}+\boldsymbol{G}_{i} \boldsymbol{G}_{i}^{T_{r}}+\frac{1}{\alpha^{2}} \hat{\boldsymbol{h}}_{i} \hat{\boldsymbol{h}}_{i}^{T_{r}}$ and $\boldsymbol{R}_{i}=\boldsymbol{F}_{i} \boldsymbol{G}_{i}^{T_{r}}$.

\subsection{Solving for the linear system}

Eq. (19) is a linear system in terms of $\overline{\boldsymbol{W}}$. In the following, using the LU factorization scheme, the sensitivity analysis problem can be simplified without need to solve all solutions in Eq.(19).

Performing block LU decomposition to the matrix of Eq.(20) yields

$$
\boldsymbol{A}=\boldsymbol{L}_{A} \boldsymbol{U}_{\boldsymbol{A}}, \boldsymbol{L}_{\boldsymbol{A}}=\left[\begin{array}{cccccc}
\boldsymbol{T}_{1} & & & & \\
\boldsymbol{R}_{1} & \boldsymbol{T}_{2} & & & \\
& \boldsymbol{R}_{2} & \mathrm{O} & & \\
& & \mathrm{O} & \boldsymbol{T}_{m-1} & \\
& & & \boldsymbol{R}_{m-1} & \boldsymbol{T}_{m}
\end{array}\right], \boldsymbol{U}_{A}=\left[\begin{array}{ccccc}
\boldsymbol{I} & \Pi_{\boldsymbol{I}} & & & \\
& \boldsymbol{I} & \Pi_{2} & & \\
& \mathrm{O} & \mathrm{O} & \\
& & \boldsymbol{I} & \Pi_{m-1} \\
& & & & \boldsymbol{I}
\end{array}\right]
$$

where $\boldsymbol{L}_{\boldsymbol{A}}$ and $\boldsymbol{U}_{\boldsymbol{A}}$ are the block lower bidiagonal matrix and block upper bidiagonal matrix, respectively and $\boldsymbol{T}_{1}=\boldsymbol{S}_{1}, \boldsymbol{T}_{i}=\boldsymbol{S}_{i}-\boldsymbol{R}_{i-1}\left(\boldsymbol{T}_{i-1}\right)^{-1} \boldsymbol{R}_{i-1}^{T_{r}}, \Pi_{i}=\left(\boldsymbol{T}_{i}\right)^{-1} \boldsymbol{R}_{i}^{T_{r}}$.

Using the block Thomas method, solving Eq. (19) becomes two relatively simpler steps: first solve $\boldsymbol{L}_{\boldsymbol{A}} \overline{\boldsymbol{X}}=\overline{\boldsymbol{b}}$ where $\overline{\boldsymbol{X}}=\left[\boldsymbol{X}_{1}^{T}, \boldsymbol{X}_{2}^{T}, \mathrm{~L}, \boldsymbol{X}_{m}^{T}\right]^{T_{r}}$, then solve $\boldsymbol{U}_{\boldsymbol{A}} \overline{\boldsymbol{W}}=\overline{\boldsymbol{X}}$. Neither steps require iteration because the left hand side matrices are bidiagonal. However, it should be noted that the relation $\boldsymbol{X}_{m}=\boldsymbol{W}_{m}$ can be derived from the last row of the block upper bidiagonal matrix $\boldsymbol{U}_{A}$ and $\boldsymbol{U}_{\boldsymbol{A}} \overline{\boldsymbol{W}}=\overline{\boldsymbol{X}}$. In addition, the sensitivity coefficient of the time averaged variable is dependent on the final state of the Lagrange multipliers $\boldsymbol{W}_{m}$. Consequently, the sensitivity coefficient is obtained as a result of solving $\boldsymbol{L}_{\boldsymbol{A}} \overline{\boldsymbol{X}}=\overline{\boldsymbol{b}}$ without requiring to solve $U_{A} \overline{\boldsymbol{W}}=\overline{\boldsymbol{X}}$.

Algorithm 1 contains a pseudocode description of the algorithm used to solve the linear equations $\boldsymbol{L}_{A} \bar{X}=\bar{b}$.

Algorithm 1. The algorithm for solving $\boldsymbol{L}_{A} \overline{\boldsymbol{X}}=\overline{\boldsymbol{b}}$

$$
\begin{aligned}
& \text { Compute } \boldsymbol{F}_{0} \\
& \text { for } i=1,2, \ldots, m \text { do }
\end{aligned}
$$

Compute $\boldsymbol{F}_{i}, \boldsymbol{G}_{i}, \hat{\boldsymbol{h}}_{i}, \boldsymbol{b}_{i}$ and $\boldsymbol{S}_{i}$

if $i=1$ 


$$
\boldsymbol{T}_{1}=\boldsymbol{S}_{1} \text { and Solve } \boldsymbol{T}_{1} \boldsymbol{X}_{1}=\boldsymbol{b}_{1}
$$

$$
\text { Compute } \boldsymbol{R}_{1}=\boldsymbol{F}_{1} \boldsymbol{G}_{1}^{T_{r}}
$$

else

$$
\begin{aligned}
& \boldsymbol{T}_{i}=\boldsymbol{S}_{i}-\boldsymbol{R}_{i-1}\left(\boldsymbol{T}_{i-1}\right)^{-1} \boldsymbol{R}_{i-1}^{T_{r}} \\
& \text { Solve } \boldsymbol{T}_{i} \boldsymbol{X}_{i}=\boldsymbol{b}_{i}-\boldsymbol{R}_{i-1} \boldsymbol{X}_{i-1} \\
& \text { Compute } \boldsymbol{R}_{i}=\boldsymbol{F}_{i} \boldsymbol{G}_{i}^{T_{r}}
\end{aligned}
$$

endif

endfor

Algorithm 1 is a nonlinear recursion formula from which the final state of the Lagrange multipliers $\boldsymbol{W}_{m}$ is determined.

The above process shows that solving the bidiagonal discretized system can be performed in a deterministic number of steps of operation, instead of being determined by whether or not meeting the convergence threshold. In other words, the combination of the proposed method and the block Thomas method is an iteration-free scheme. This method will not have convergence issue .

Moreover, it can be seen that solving a discretized linear system $\boldsymbol{L}_{A} \overline{\boldsymbol{X}}=\overline{\boldsymbol{b}}$ of the proposed method is a series of subdomain based matrix calculations. Compared to solving a huge global system of matrix equation $\boldsymbol{A} \overline{\boldsymbol{W}}=\overline{\boldsymbol{b}}$ by the LSS method, the proposed method treats a bunch of matrix equations with much smaller sizes. Furthermore, the LSS method solves all solutions of linear equations simultaneously in comparison with the developed method that only needs to keep the solutions in one or two previous time levels.

Generally speaking, the computational complexity and memory consumption of inverting or factorizing a block tridiagonal type sparse matrix $\boldsymbol{A}$ increase rapidly with respect to the corresponding number of time steps and system dimension. Due to this reason the proposed method is more promising than the LSS method in solving large scale problems.

The proposed method requires the storage or recomputation of the inverse matrice of $\boldsymbol{T}_{i}$ in Eq.(21) which either increases the storage cost or the computational time. To reduce this cost, the iteration method using some preconditioner $\boldsymbol{P}$ may be employed as follows.

$$
\begin{aligned}
& \boldsymbol{P} \Delta \boldsymbol{x}=\left(\boldsymbol{b}_{i}-\boldsymbol{R}_{i-1} \boldsymbol{X}_{i-1}\right)-S_{i} \boldsymbol{x}_{k}+\boldsymbol{\alpha}, \\
& \boldsymbol{x}_{k+1}=\boldsymbol{x}_{k}+\Delta x
\end{aligned}
$$


where $\boldsymbol{\alpha}=\boldsymbol{R}_{i-1}\left(\boldsymbol{T}_{i-1}\right)^{-1} \boldsymbol{R}_{i-1}^{T_{r}} \boldsymbol{x}_{k}$ and $\boldsymbol{x}_{k}$ is the vector value of $\boldsymbol{X}_{i}$ after $k$ iterations. For computing $\boldsymbol{\alpha}, \boldsymbol{y}_{k}=\boldsymbol{R}_{i-1}^{T_{r}} \boldsymbol{x}_{k}$ is firstly calculated. Then solve $z_{k}$ iteratively from $\boldsymbol{T}_{i-1} \boldsymbol{z}_{k}=\boldsymbol{y}_{k}$. Finally, $\boldsymbol{\alpha}=\boldsymbol{R}_{i-1} z_{k}$ is obtained.

\subsection{Summary of the algorithm}

Based on the methods described in section 5.1 and 5.2, the proposed sensitivity analysis algorithm can be summarized as follows:

(1)Give an initial value of $\boldsymbol{U}_{0}$ at an initial time $t=t_{0}$ and the time step size $\Delta t$. Solve the augmentation equations in Eq.(4) forward in time $t \in\left[t_{0}, t_{0}+T\right]$ yielding $\boldsymbol{U}_{i}(i=0,1, \ldots, m)$;

(2)Solve the linear equation in Eq.(19) for $\boldsymbol{W}_{m}$ using algotihm 1 with $\boldsymbol{U}_{i}$.

(3) Using $\boldsymbol{G}_{m}$ and $\boldsymbol{W}_{m}$, the sensitivity of $\boldsymbol{V}_{m}$ can be calculated according to Eq.(18).

In this section, an improvement to the LSS method is proposed based on the augmented equations in Eq.(4). The improved method is an extension and also modification of LSS scheme. An explicit expression for the sensitivity coefficient is derived which lets to calculate the sensitivity coefficients in a simply way. The main advantages of the sensitivity analysis scheme are its simplicity and independency of $\boldsymbol{V}_{i}(i=0,1,2, \ldots m-1)$ and $\overline{\boldsymbol{\eta}}$ which makes the numerical sensitivity analysis presented here very accurate and efficient.

Note that the least square shadowing algorithm requires that the solutions for $\boldsymbol{V}_{i}, \eta_{i}$ and $\boldsymbol{W}_{i}$ at each time step should be calculated and stored. The time cost and the memory occupancy of the LSS method increase quickly as increasing the time steps. For lack of computer memory, it might be impossible to store these vectors and related matrices at a sufficiently high number of time instances. By contrast, the developed method can avoid having to compute the full time histories of $\boldsymbol{V}(t)$ and $\eta(t)$. In addition, there is no need to store the full solution history of the Lagrange multipliers since the final value $\boldsymbol{W}_{m}$ is required to calculate the sensitivities. Therefore, the computational cost and memory requirements for the sensitivity analysis can be significantly reduced for problems involving a large number of time steps.

\section{Sensitivity analysis results}

In this section, the effectiveness of the proposed method is illustrated through three numerical examples. The benchmark examples in the literature are utilized to verify the 
proposed method. Numerical results are initially presented for a scalar nonlinear ordinary differential equation, the Van der Pol's equation. The validation of the proposed method via the Lorenz system is then given. After that an aero-elastic limit cycle oscillator undergoing self-induced chaos oscillation is focused upon.

\subsection{Numerical results for the Van der Pol oscillator}

In order to illustrate the developed direct differentiation method, the sensitivity problem is solved for the classic Van der Pol oscillator and the results are compared with those obtained with the LSS method.

The Van der Pol oscillator studied is written as

$$
\frac{d^{2} y}{d t^{2}}=-y+\beta\left(1-y^{2}\right) \frac{d y}{d t}
$$

where $\beta$ is the influence parameter studied.

Similar to Wang [21], the response function is considered as $[e(t)]^{1 / 8}$ where $e(t)=\frac{1}{t} \int_{0}^{t} J\left(\boldsymbol{u}\left(t^{\prime}\right), s\right) d t^{\prime}, J(\boldsymbol{u}(t), s)=\left(\frac{d y(t)}{d t}\right)^{2}$ and $\boldsymbol{u}(t)=\left[y(t), \frac{d y(t)}{d t}\right]^{T_{r}}$. The derivative of $[e(t)]^{1 / 8}$ with respect to $\beta$ can be derived as $\frac{d[e(t)]^{1 / 8}}{d \beta}=\frac{-[e(t)]^{7 / 8}}{8} \frac{d[e(t)]}{d \beta}$. Obviously, $\frac{d[e(t)]}{d \beta}$ can be calculated by the direct differentiation method in Eq.(6).

Numerical simulations are computed by the fourth-order Runge-Kutta scheme with fixed step size 0.02 . The integration time for the motion and sensitivity equations is set from $t 0=1 \mathrm{e}-$ 10 to $T=5000$ to maintain stable computation of the analytical sensitivity. The portraits of $y(t)$ and the time histories of the time averaged variable $e(t)$ for different values of $\beta$ are depicted in Fig.2. To illustrate the solutions for $e(t)$ in detail, the magnification of plots is also presented in Fig.3. 


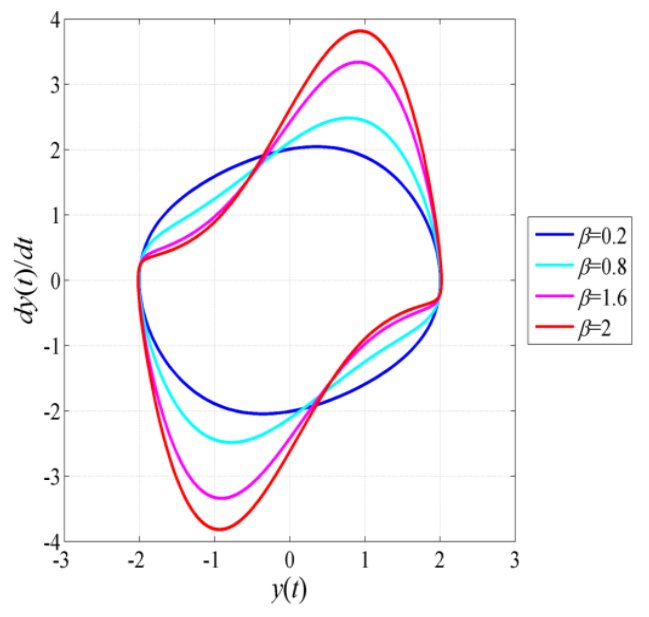

(a)The portraits of $y(t)$

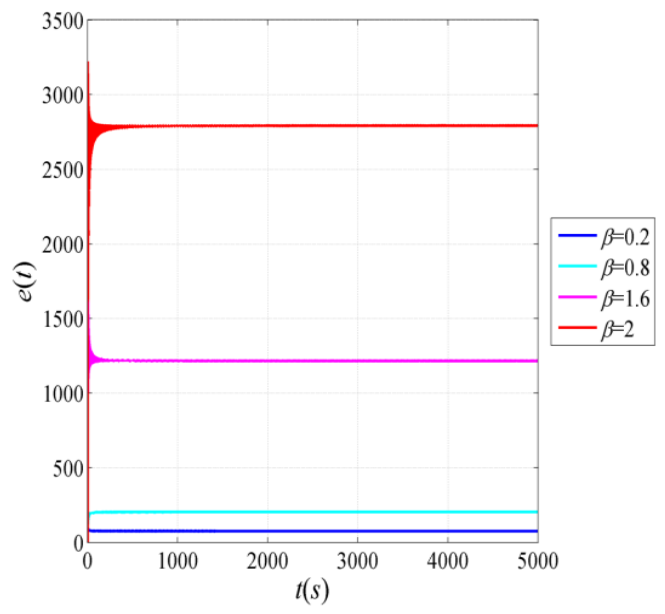

(b)The histories of $e(t)$

Fig.2 Dynamical behavior of the Van der Pol oscillator for different values of $\beta$

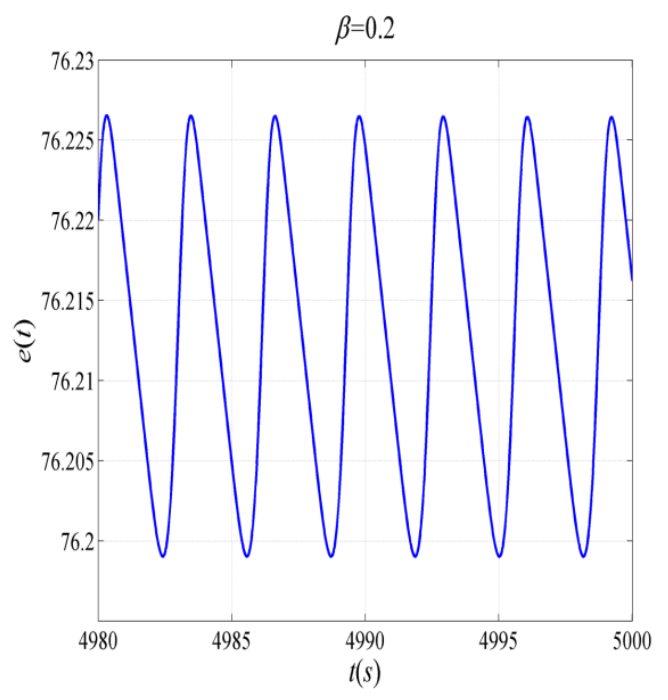

(a)

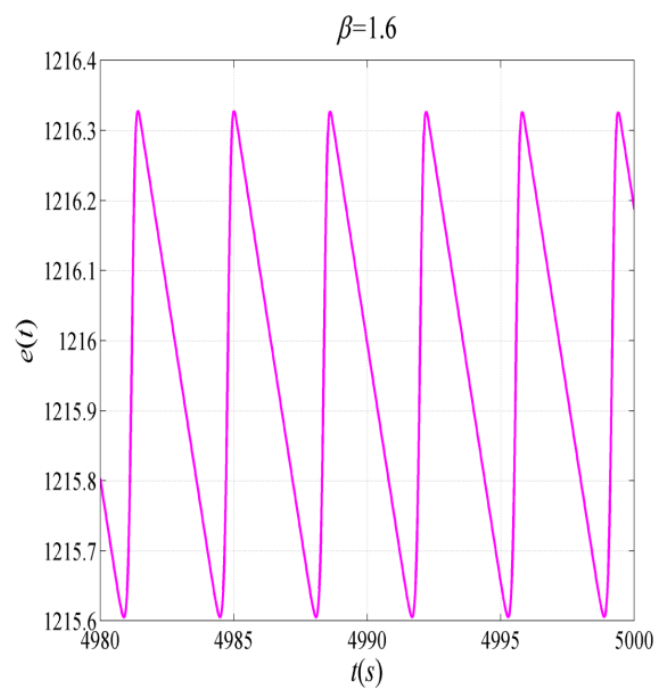

(c)

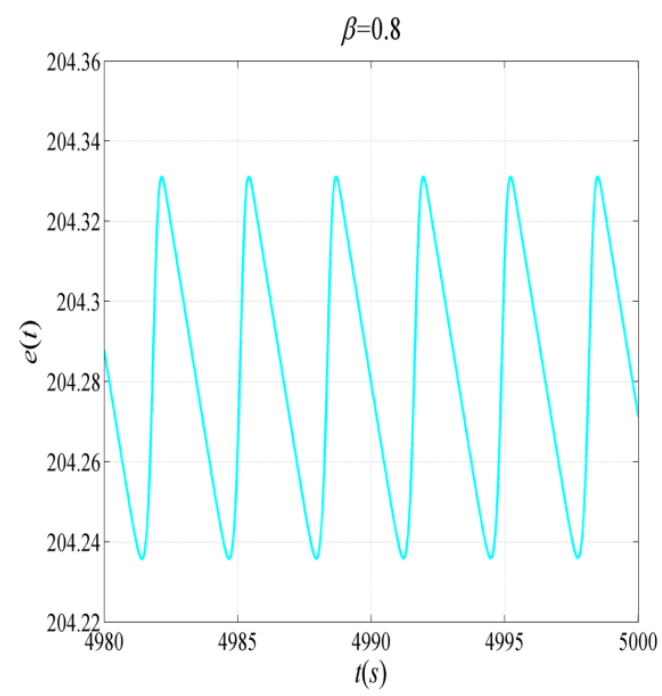

(b)

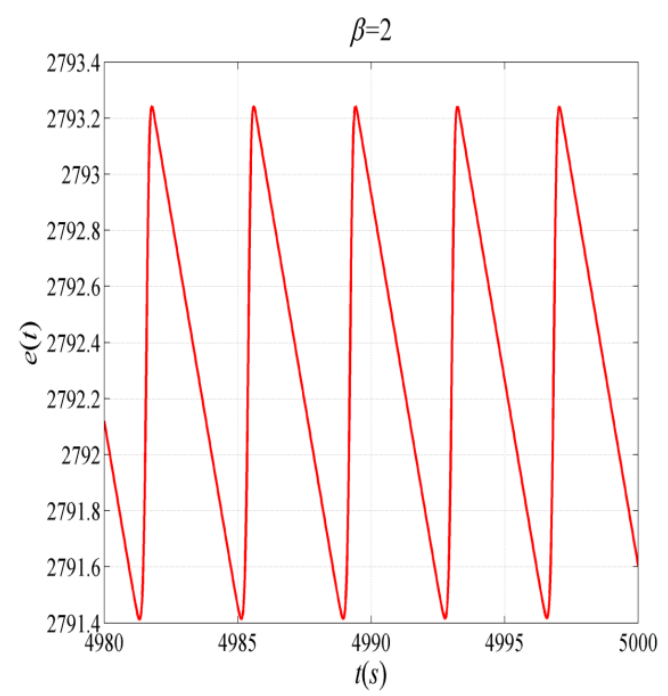

(d)

Fig.3 Zoom in parts for the solutions in Fig.2(b) 
As illustrated in Fig.2, the displacement $y(t)$ for the four cases is acting with periodic motions and the numerical results in Fig.2 indicate that the time averaged variable $e(t)$ keeps constant. On the contrary, it can be clearly seen from the partial enlarged plots shown in Fig.3 that the solutions for $e(t)$ contain repeating patterns in time.

The sensitivity results for different $\beta$ values corresponding to the solutions indicated in Fig.2 are illustrated in Fig.4. In order to allow more detailed display of the sensitivity variation, some parts in these figures are magnified in Fig.5.

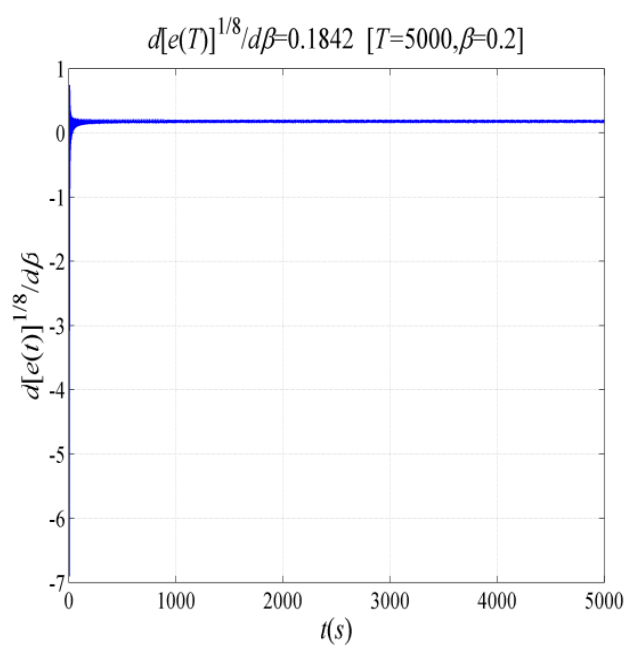

(a) $\beta=\mathbf{0 . 2}$

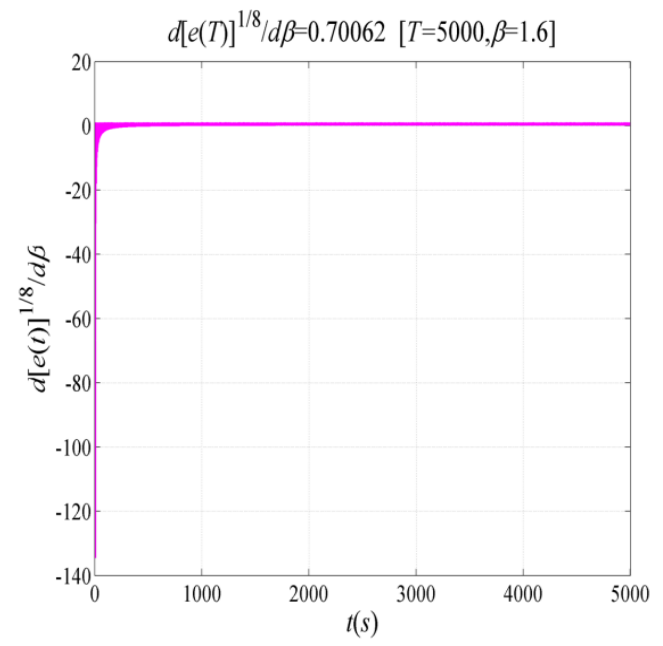

(c) $\beta=1.6$

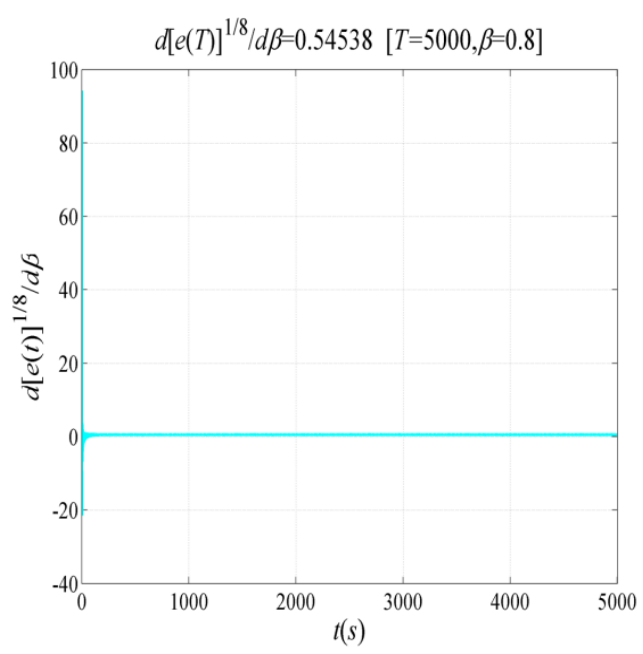

(b) $\beta=0.8$

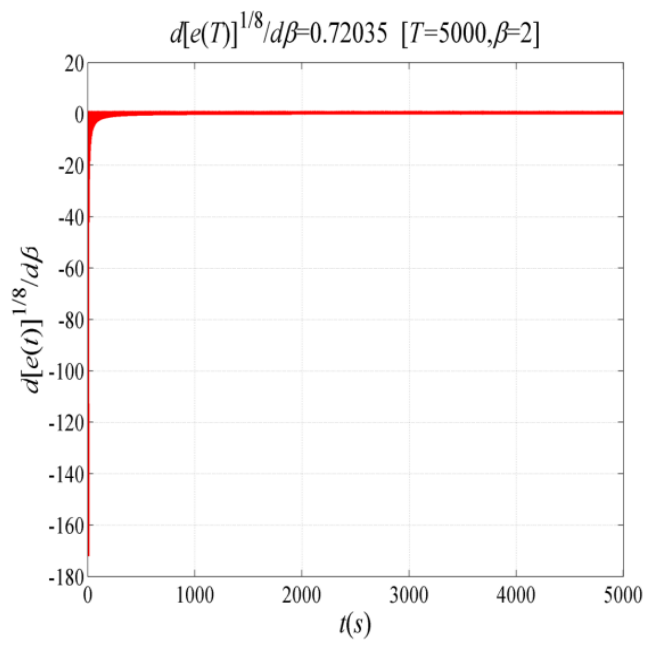

(d) $\beta=\mathbf{2}$

Fig. 4 The sensitivity coefficients of different $\beta$ obtained by using the direct differential method 


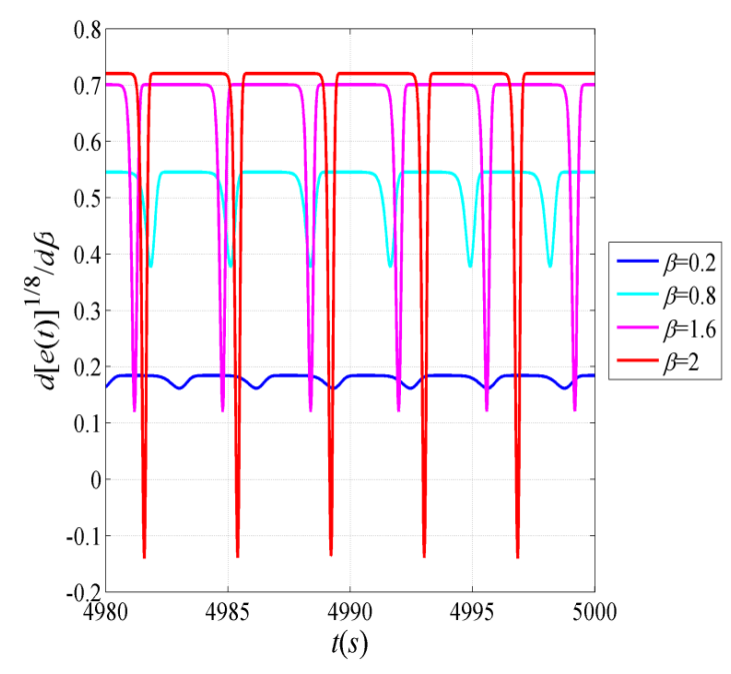

Fig. 5 Zoom in parts showing the sensitivity results in Fig.4

As shown in Figs.4 and 5, the same phenomena that the sensitivity history shows a fluctuating behavior around its constant value is visible. For $\beta=2$, the sensitivity is altered in $[-0.15,0.72035]$ and a maximum value of 0.72035 is produced by the system. As it can be observed from these figures, the magnitude of the sensitivity spikes is increased and the duration of the local sensitivity coefficients is decreased as the parameter value of $\beta$ is increased.

The direct differentiation method is repeatedly applied for $\beta$ in the range of $[0.2,2]$ with step size 0.1 and the constant value of the sensitivity coefficient around $T=5000$ for each $\beta$ is used to plot the sensitivity curve. The sensitivity results are depicted in Fig.6. Looking at the sensitivity coefficients in Fig.6, it is observed that the sensitivity value of $e(t)$ with respect to $\beta$ increases monotonically with the increase of $\beta$ and reaches the maximum at the upper limit of $\beta$. When compared with the results of Wang[21], the curve in Fig.6 is in agreement with the results in Fig.4(d) of [21] and the overall shape of the direct differentiation method and the LSS method matches well with each other. 


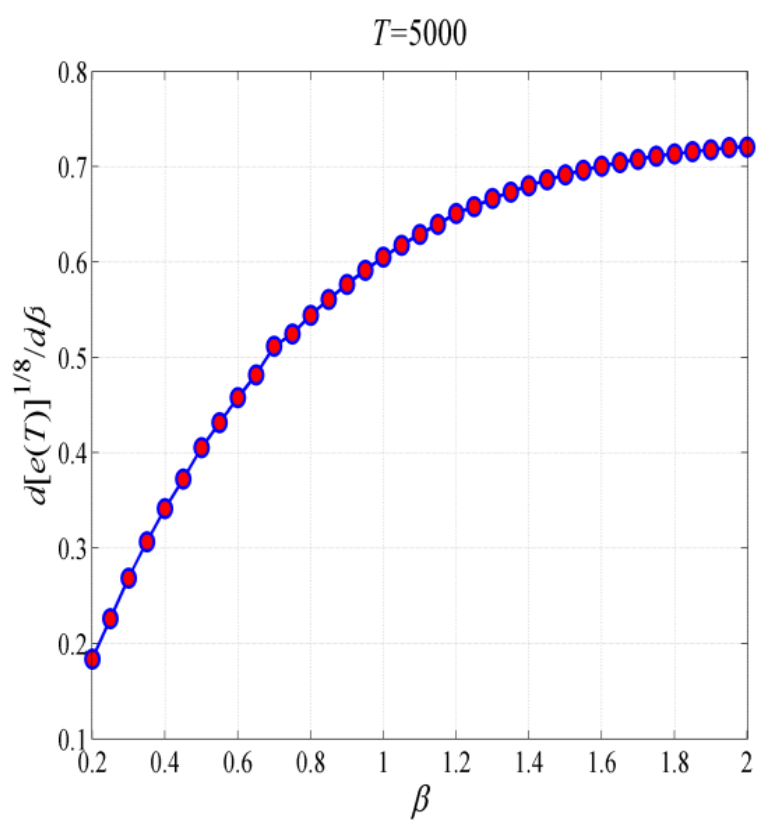

Fig. 6 The sensitivity coefficients as a function of $\beta$ obtained by the direct differentiation method

This simple example illustrates the advantage of the proposed direct differentiation method that shows the ability to predict the accurate sensitivity coefficients of nonlinear systems.

\subsection{Numerical results for the Lorenz system}

As the second verification example, Lorenz's oscillator which is a 3 dimensional dynamical system that exhibits chaotic flow is selected. The Lorenz system is described as:

$$
\begin{aligned}
& \frac{d x}{d t}=\sigma(y-x) \\
& \frac{d y}{d t}=x(\rho-z)-y \\
& \frac{d z}{d t}=x y-\beta z
\end{aligned}
$$

where $\sigma$ is called the Prandtl number and $\rho$ represents the Rayleigh number.

Numerical examples which have been taken from the publication by Wang[21] are considered with the following parameters $\sigma=10$ and $\beta=8 / 3$. Through the analysis of the dynamic characteristic in [21], it can be noted that the system meets chaotic status when $\rho$ increases through 24.06 and non hyperbolic strange attractors persist for most of the range of $31<\rho<99.5$. In contrast, the Lorenz system exhibits quasi hyperbolic strange attractors when the Rayleigh number $\rho$ is in some certain parameter range $(24.06<\rho<31)$.

The time averaged variable is chosen as $e(t)=\frac{1}{t} \int_{0}^{t} z\left(t^{\prime}\right) d t^{\prime}$. Resembling the previous numerical analysis in section 6.1, the direct differentiation method is firstly used to calculate the sensitivity of $e(t)$ with respect to $\rho$ for five distinct cases: $\rho=10, \rho=25, \rho=50, \rho$ $=75$ and $\rho=100$. 
The time integration solutions for different values of $\rho$ are displayed in Fig.7. Fig.8 shows zoomed-in sections of the signals presented in Fig.7(b).From Fig.7(a) it is noticed that the system for the three cases of $\rho=25, \rho=50$ and $\rho=75$ displays chaotic motions while fixed point attractor and limit cycle attractor are found for $\rho=10$ and $\rho=100$, respectively. As shown in Fig.8, the waveform has no oscillation for $\rho=10$. On the contrary, the system shows random(chaotic) behavior for $\rho=25, \rho=50$ and $\rho=75$. The periodic behavior of the system at $\rho=100$ is noted in Fig.8(e) and the amplitude has notably larger value.

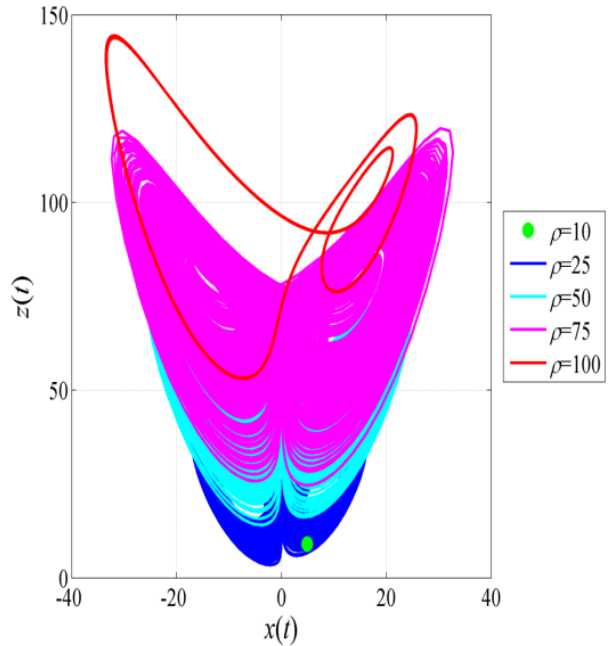

(a)The attractors

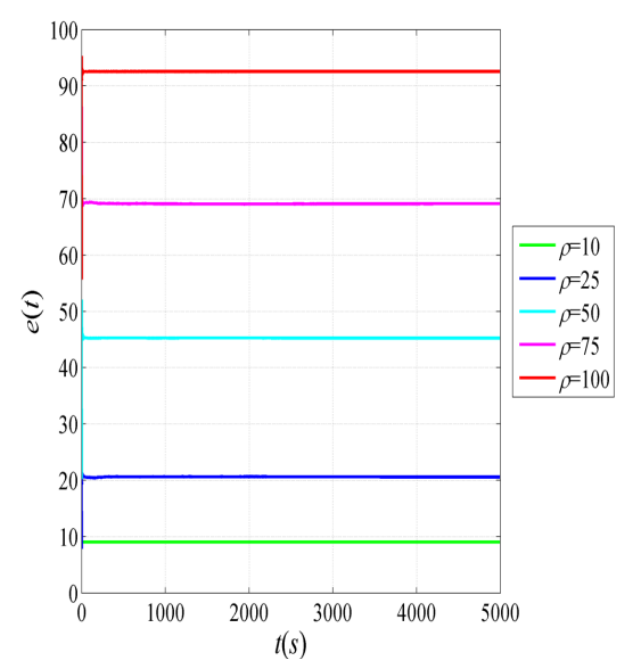

(b)The histories of $e(t)$

Fig.7 The time integration solutions for different values of $\rho$

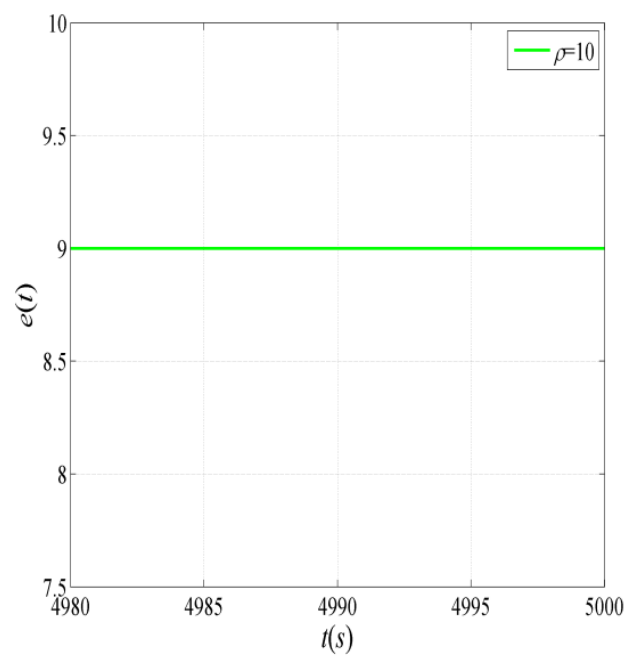

(a)

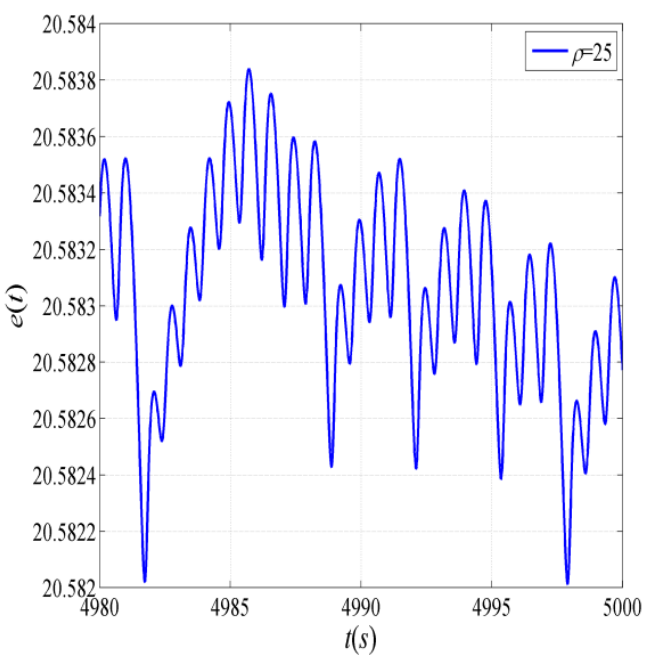

(b) 


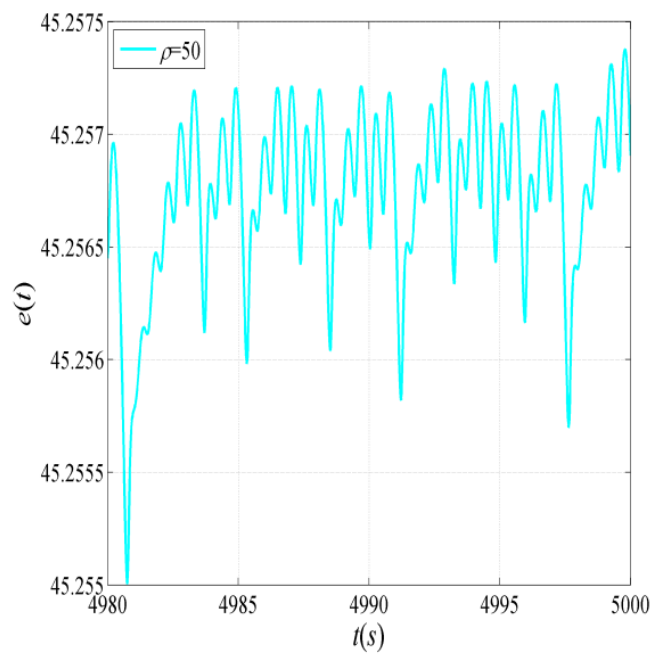

(c)

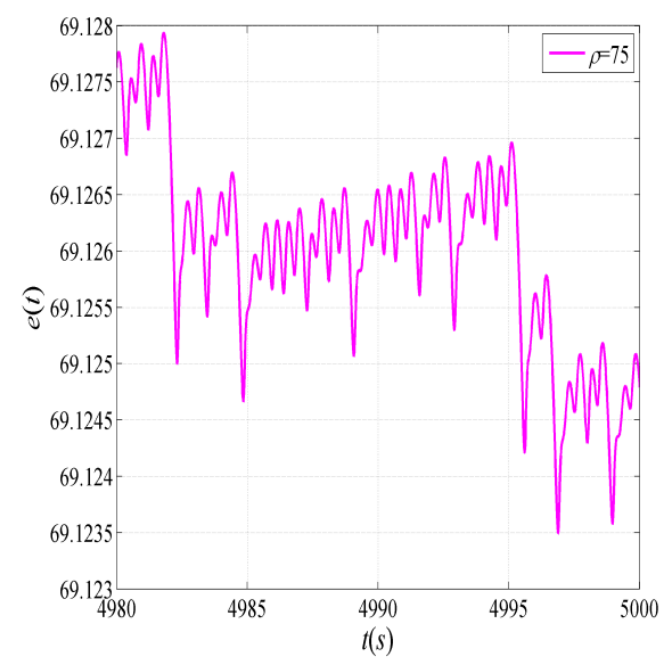

(d)

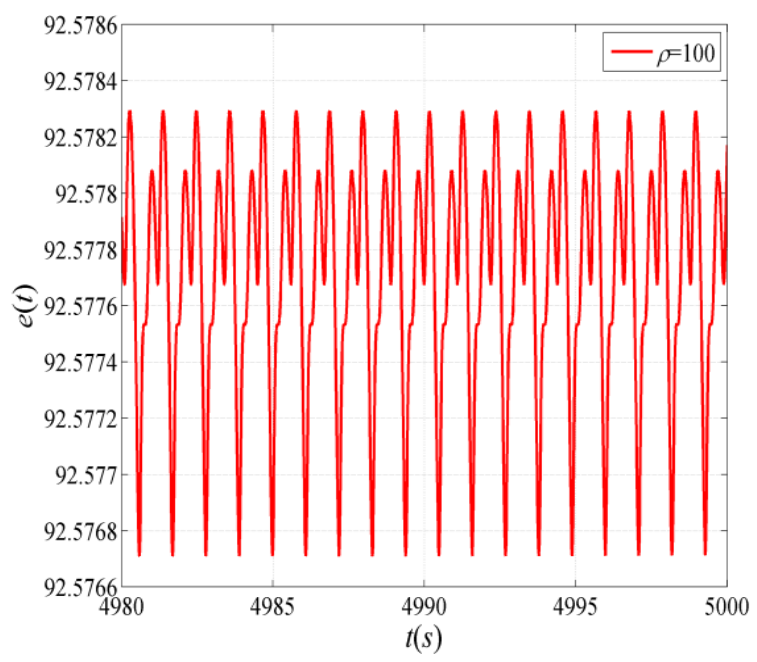

(e)

Fig. 8 Zoom in parts showing the results in Fig.7(b)

Based on the direct differentiation method, the sensitivity results for the two cases of $\rho$ $=10$ and $\rho=25$ are illustrated in Fig.9. As illustrated in Fig.9, the sensitivity coefficients for $\rho=10$ become an ordinary constant which is equal to 1 . In contrast, the sensitivity amplitude in Fig.9(b) follows the asymptotic line perfectly and the sensitivity coefficients for $\rho=25$ are divergent. Similar divergent sensitivity coefficients for the cases of $\rho=50$ and $\rho=75$ are also obtained and not presented here. 


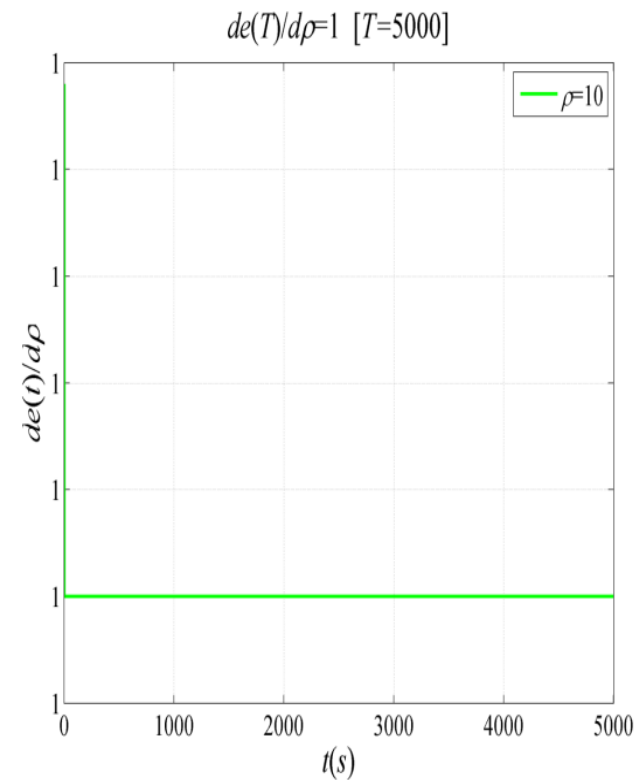

(a)

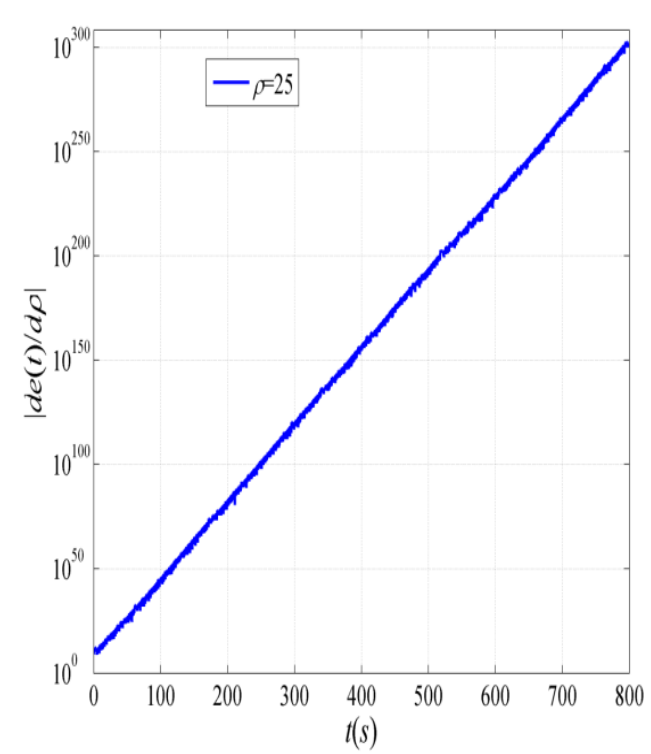

(b)

Fig. 9 The time histories of the sensitivity coefficients for different $\rho$ obtained by the direct

\section{differentiation method}

It should be noticed that the integration of this chaotic system for the cases of $\rho=25, \rho$ $=50$ and $\rho=75$ leads to numerically unstable solutions(i.e numerical explosions) even with a very small time step and the sensitivity coefficients will increase in time due to the usual sensitivity to initial conditions of chaotic system. This evidences that the direct differentiation method is not suitable in this scenario. Instead, more sophisticated method like the improved least squares shadowing method presented seems to be a proper choice.

Before applying the improved LSS method, the trajectory $\boldsymbol{U}(t)$ on the attractor should be calculated. The trial numerical simulation in which initial conditions with $[0,1]$ uniform numbers are assigned is firstly performed so that the final state of $\boldsymbol{U}(t)$ (such as $t=50$ for this example) is approximately on the attractor. By utilizing the final state values in the previous trial simulation as initial conditions, the solution $\boldsymbol{U}(t)$ over the time interval $\left[t_{0}, t_{0}+T\right]$ is calculated and used for further sensitivity analysis. Numerical tests are carried out with a constant step size $\Delta t=0.01$. The integration time from $t_{0}=1 \mathrm{e}-10$ to $t_{0}+T$ with $T=5000$ is introduced to perform a stable calculation of the sensitivity.

The numerical sensitivity results obtained from the improved LSS method for the five cases of $\rho$ are summarized in Table 1 where the Lagrange multipliers evaluated at $T$ are also listed. As can be seen in Table 1, the derivative for $\rho=10$ is 0.9999 and a comparison of the sensitivity values obtained with the improved LLS method and the direct differentiation method for $\rho=10$ shows a good agreement. The prediction of the sensitivity value for $\rho=10$ is fairly accurate.

Table 1 Summaries of sensitivity coefficients for the Lorenz oscillator.

\begin{tabular}{cccccc}
\hline$\rho$ & 10 & 25 & 50 & 75 & 100 \\
\hline $\boldsymbol{W}_{m}$ & 0.0028 & 0.0014 & -0.0025 & $1.7214 \mathrm{e}-4$ & -0.0017
\end{tabular}




\begin{tabular}{cccccc} 
& 0.0031 & 0.0038 & -0.0046 & 0.0024 & -0.0052 \\
& 0.0098 & 0.0028 & 0.0017 & 0.0017 & 0.0068 \\
& 0.0100 & 0.0103 & 0.0099 & 0.0098 & 0.0118 \\
\hline$\frac{d[e(T)]}{d \rho}$ & 0.9999 & 1.0258 & 0.9880 & 0.9803 & 1.1846
\end{tabular}

In order to test the robust of the improved LSS method, 20 samples with $T=50$ for each value of $\rho$ are used to find for the statistical distributions of the sensitivity coefficients. The time averaged variable $e(t)$ and its sensitivities evaluated at $T$ are depicted in Fig.10 where notations ' $T=50$ ' and ' $T=5000$ ' represent the sensitivity coefficients computed by the time integration solutions of length $T=50$ and $T=5000$, respectively.

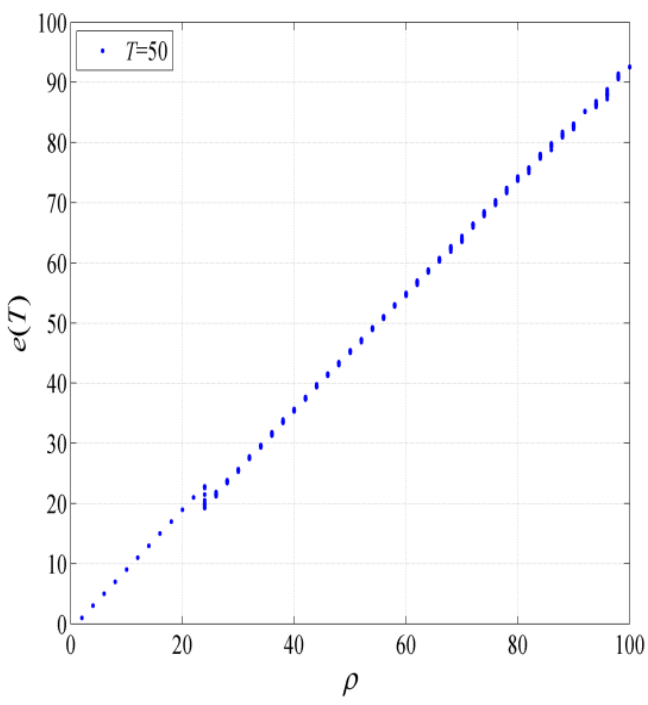

(a)

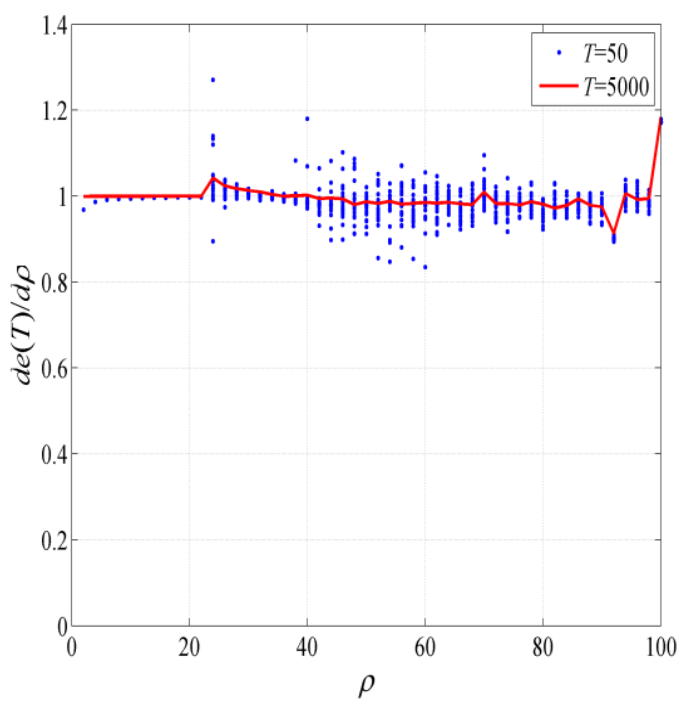

(b)

Fig. 10 The time averaged quantities and its sensitivity coefficients for two cases of system trajectory length. The blue dots represent the numerical results related to the time integration solutions of length 50.The red line indicate the numerical results for the time integration solutions of length 5000 .

It can be seen from Fig.10 that the sensitivity values of the 20 sample distributions are very close to the sensitivity values of $T=5000$ when the flow velocity is less than 22 . Within this parameter range, the airfoil system shows periodic motions. Moreover, some sudden fluctuations in these sensitivities are noted at $\rho=24$ and relatively larger deviation of the sensitivity distribution is detected for the flow velocity between 40 and 60 . In addition, the sensitivity for the case of $T=5000$ reaches a maximum value at the upper limit of $\rho$.

Turning back on a comparison with Wang [21], the numerical results in Fig.10 are extremely similar to the ones obtained in Fig.5 of [21]. The sensitivity results between the improved LSS method and the original LSS method agree satisfactorily with each other. It should be emphasized that the predictions of sensitivity efficient by using the improved least 
squares shadowing approach are also efficient for non hyperbolic attractors which exist for most of the parameter range considered $(31<\rho<99.5)$.

To represent the developed approach precisely and qualitatively, the sensitivity coefficients are examined with parameter fixed as $\rho=28$. The sensitivity coefficients resulted from 200 samples for two cases of $T$ are illustrated in Figs.11 and 12. Looking at the results in Figs.11 and 12, it is obvious that the fluctuation ranges of the sensitivity coefficients for $T=5000$ are narrower than that for $T=1000$. The longer the time length $T$ is, the more accurately the sensitivity coefficients are. The overall quality of the prediction is improved by using larger $T$.

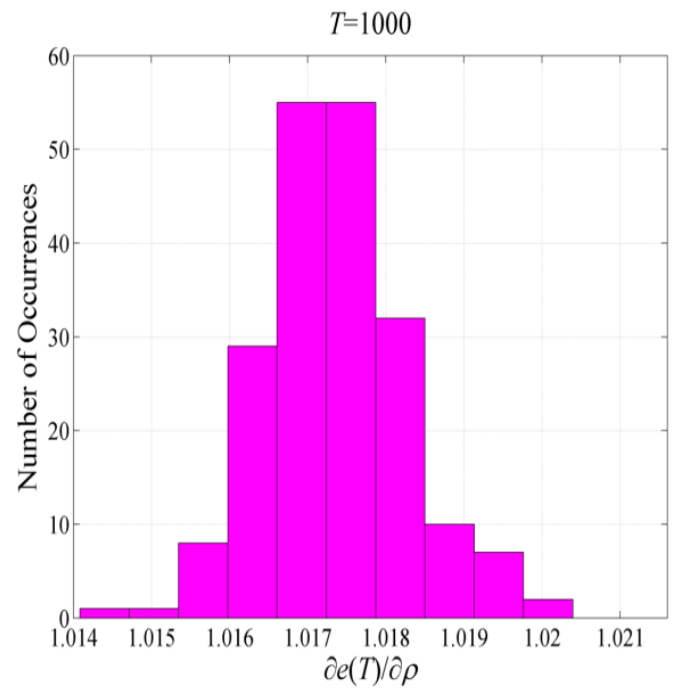

(a)

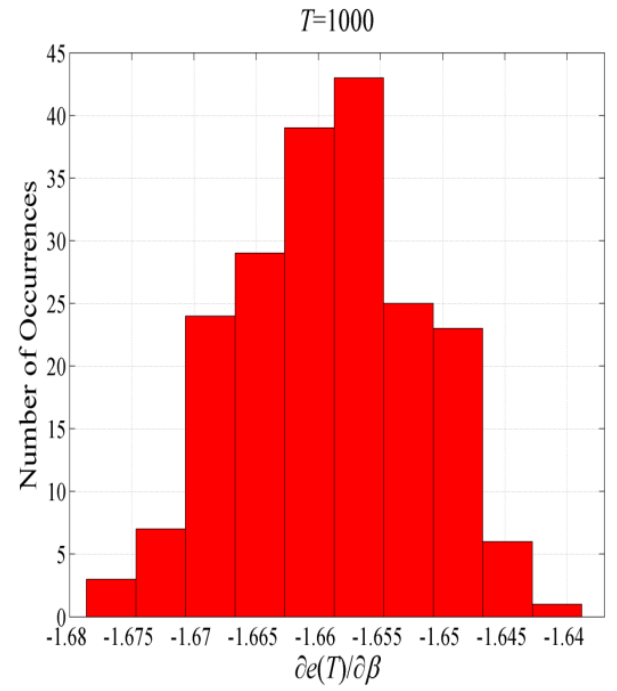

(b)

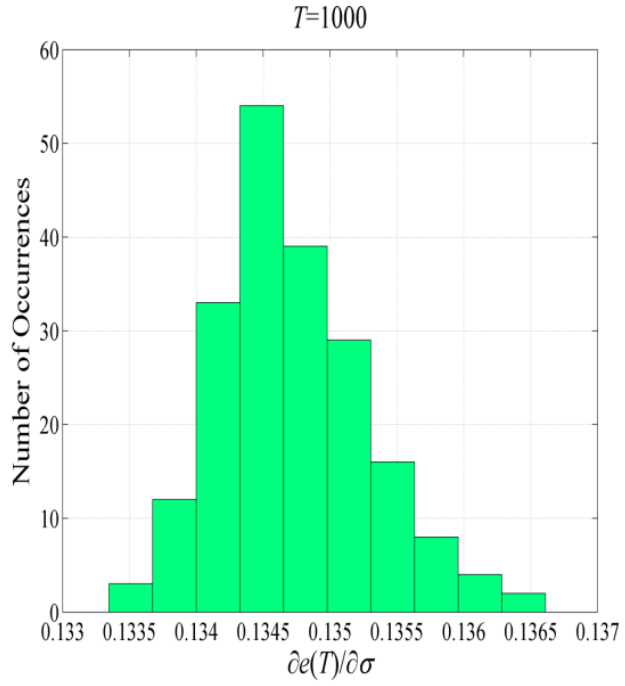

(c)

Fig.11 The statistic information of the sensitivity coefficients for $T=1000$ 


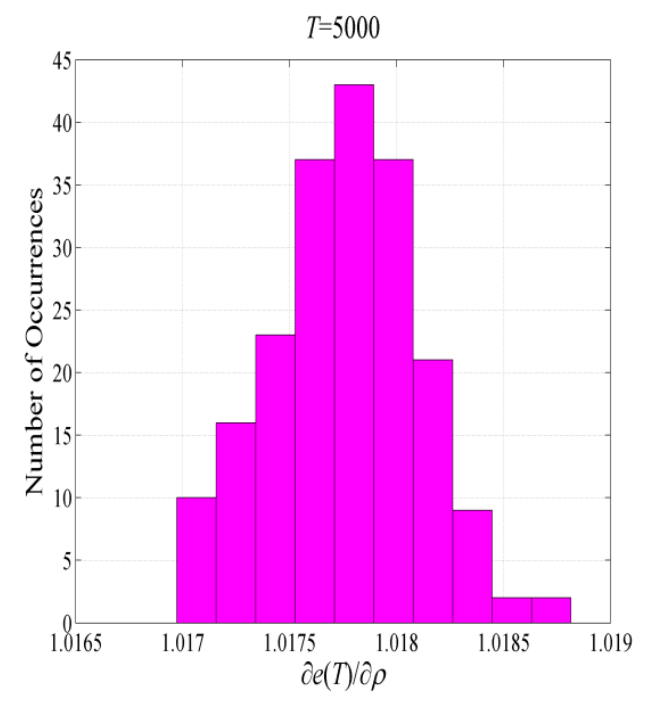

(a)

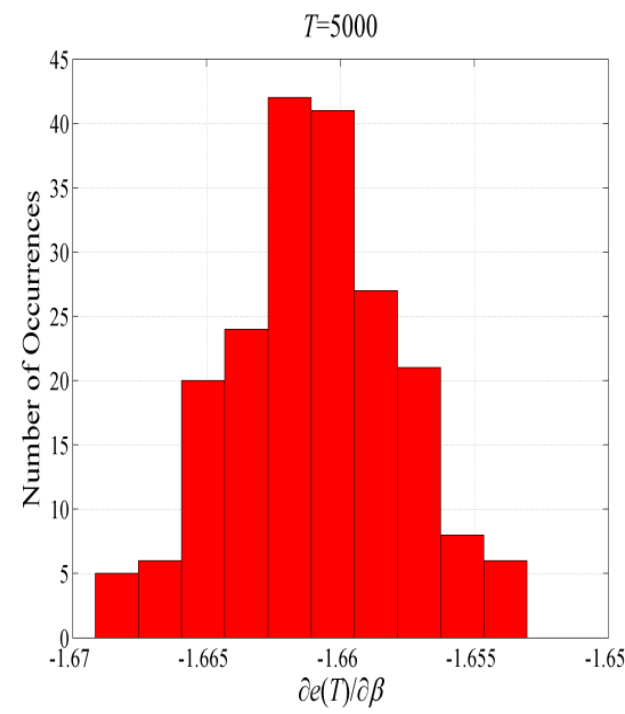

(b)

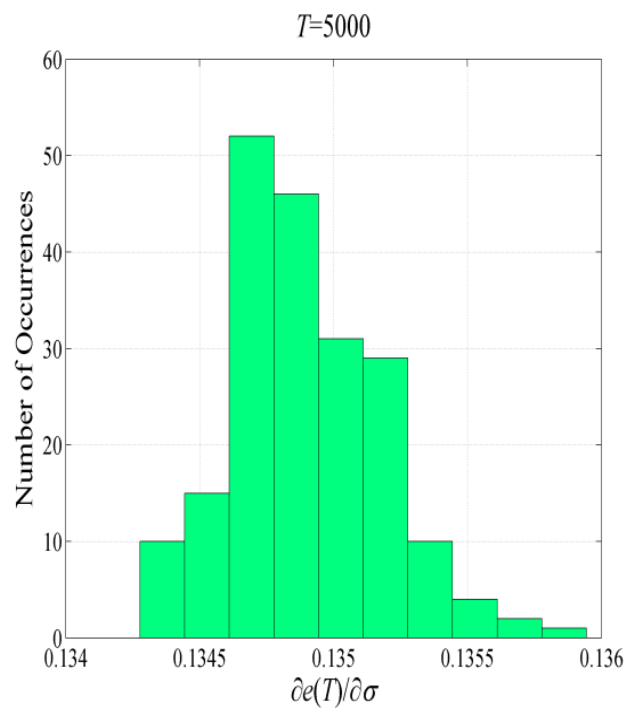

(c)

Fig.12 The statistic characteristics of the sensitivity coefficients for $\boldsymbol{T}=\mathbf{5 0 0 0}$

In the following, the numerical results in Figs.11 and 12 are compared with the results in [20][26]. For $T=1000$ in Fig.11, the sensitivity values of 200 samples are altered in the ranges $\frac{\partial[e(T)]}{\partial \rho} \in \quad[1.0141,1.0204], \quad \frac{\partial[e(T)]}{\partial \beta} \in \quad[-1.6786,-1.6388]$, and $\quad \frac{\partial[e(T)]}{\partial \sigma} \epsilon$ [0.1333,0.1366],respectively. The counterparts in Fig.12 for $T=5000$ are within the intervals $\frac{\partial[e(T)]}{\partial \rho} \in[1.0170,1.0188], \frac{\partial[e(T)]}{\partial \beta} \in[-1.6692,-1.6530]$ and $\frac{\partial[e(T)]}{\partial \sigma} \in[0.1343,0.1359]$. By comparing the linear regression results in Eq.(46) of [20] which predicts the sensitivity coefficients $\frac{\partial[e(T)]}{\partial \rho}=1.01 \pm 0.04, \frac{\partial[e(T)]}{\partial \beta}=-1.68 \pm 0.15, \frac{\partial[e(T)]}{\partial \sigma}=0.16 \pm 0.02, \quad$ the 
proposed method under predicts $\frac{\partial[e(T)]}{\partial \sigma}$ while for $\frac{\partial[e(T)]}{\partial \rho}$ and $\frac{\partial[e(T)]}{\partial \beta}$ the two approaches give almost the same results. It should be noted that the Lyapunov eigenvector decomposition method reports the sensitivity results in Fig.9 and Eq.(45) of [20], which gives $\frac{\partial[e(T)]}{\partial \rho}=0.97, \frac{\partial[e(T)]}{\partial \beta}=-1.74$ and $\frac{\partial[e(T)]}{\partial \sigma}=0.21$. Similarly, the sensitivity coefficients $\frac{\partial[e(T)]}{\partial \rho}=1.00, \frac{\partial[e(T)]}{\partial \beta}=-1.67$ and $\frac{\partial[e(T)]}{\partial \sigma}=0.122$ are obtained in Eq.(10) of [26] by using the LSS method with multi grid solvers. Qualitatively comparisons of the sensitivity results obtained from different methods demonstrate that good agreements of these approaches to estimate the sensitivity coefficients are achieved although small discrepancies are observed, which confirm that the proposed algorithm is accurate and efficient.

6.3 Numerical results for an aero-elastic limit cycle oscillator

An airfoil system is adopted here as the third example and shown in Fig.13[21][4].The plunge deflection is denoted by $h$, which is positive in the downward direction, and $\alpha$ is the pitch angle between the elastic axis and the positive nose up direction.

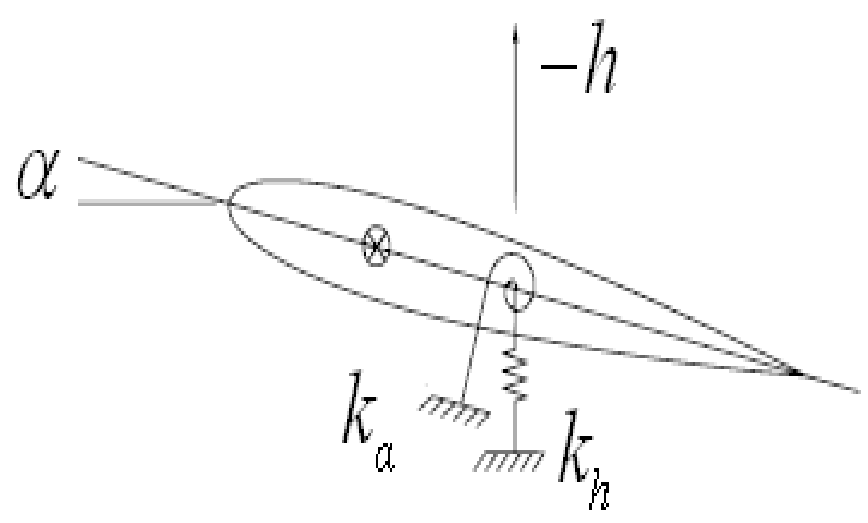

Fig.13 Schematic diagram of the airfoil system

The aeroelastic motion of the 2-dof airfoil is governed by the following coupled bendingtorsion equation:

$$
\mathbf{M} \ddot{\boldsymbol{x}}+\mathbf{D} \dot{\boldsymbol{x}}+\left(\mathbf{K}_{1}+\mathbf{K}_{2} Q\right) \boldsymbol{x}+\mathbf{K}_{3} \boldsymbol{x}^{3}=0
$$

where $\mathbf{M}, \mathbf{D}$ and $\mathbf{K}_{\mathbf{1}}, \mathbf{K}_{\mathbf{2}}, \mathbf{K}_{\mathbf{3}}$ are respectively the generalized mass, damping, and stiffness matrices and $\boldsymbol{x}=\left\{\begin{array}{l}h \\ \alpha\end{array}\right\}, \mathbf{K}_{3}=\left[\begin{array}{cc}k_{h} & 0 \\ 0 & k_{\alpha}\end{array}\right] \cdot k_{h}, k_{\alpha}$ are the nonlinear plunge and pitch cubic stiffness coefficient, respectively. $Q$ denotes the flow speed.

The structural matrices which are the same with that of [21] have the following form: 


$$
\mathbf{M}=\left[\begin{array}{cc}
1 & 0.25 \\
0.25 & 0.5
\end{array}\right], \mathbf{D}=\left[\begin{array}{cc}
0.1 & 0 \\
0 & 0.1
\end{array}\right], \mathbf{K}_{1}=\left[\begin{array}{cc}
0.2 & 0 \\
0 & 0.5
\end{array}\right], \mathbf{K}_{2}=\left[\begin{array}{cc}
0 & 0.1 \\
0 & -0.1
\end{array}\right]
$$

The bifurcation diagram of the system using the flow velocity $Q$ as the control parameter by numerical simulation has been analyzed in Fig.8(a) of [21]. As shown in Fig.8(a) of [21], a transition from period motion to chaotic behavior, and finally back to period motion is seen when $Q$ is increased from 8 to 16 . Specifically, the system response enters into the chaotic region from 11.2 to 12.4 .

Time histories and phase portraits are constructed for two cases of $Q$ (11 and 12) to assess the system behavior. Fig.14 shows the time history plot and phase portrait for $Q=11$. The results are plotted only for simulation time between 29900 and $30000 \mathrm{~s}$. It can be seen from Fig.14 that the orbit takes the form of a closed curve consisting of four interlocked loops in the phase-space and the periodic motion is observed.

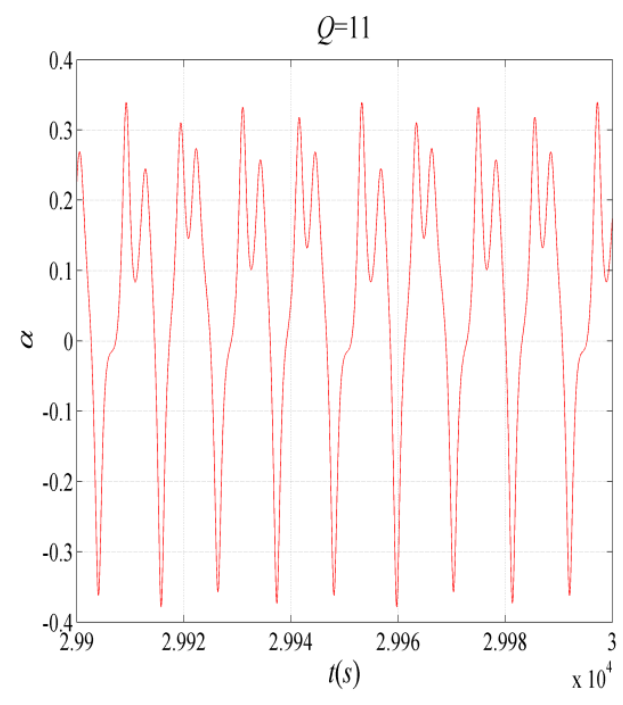

(a)Time history plot

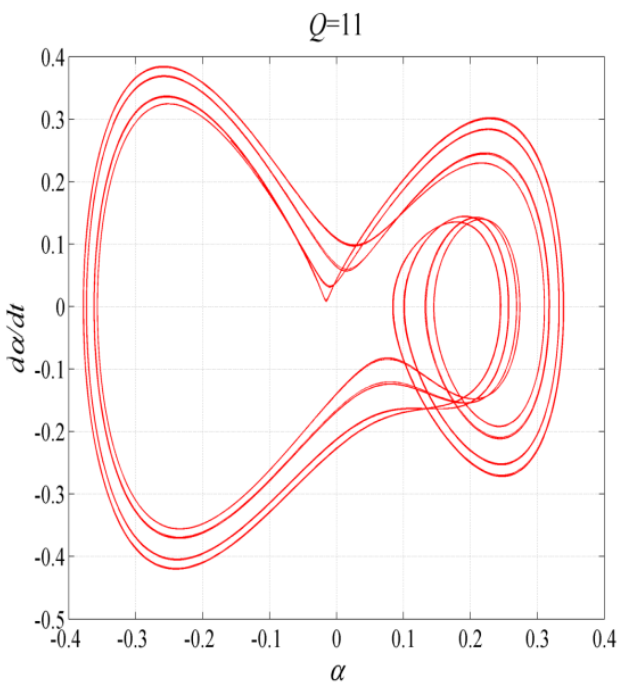

(b)Phase portrait

Fig.14 Time history plot and phase portrait of the airfoil system at $Q=11$

The time series and phase portraits of the airfoil system at $Q=12$ are presented in Figs.15 and 16. As demonstrated in Fig.15, complex motions(such as chaos and chaotic transition) take place. The transition of the airfoil motion from chaos to the periodic state can be observed clearly in Figs.15(b)and 16. Chaotic behavior is visible for $t<2900$. But when $t>2900$, the system transits from chaotic to periodic state. 


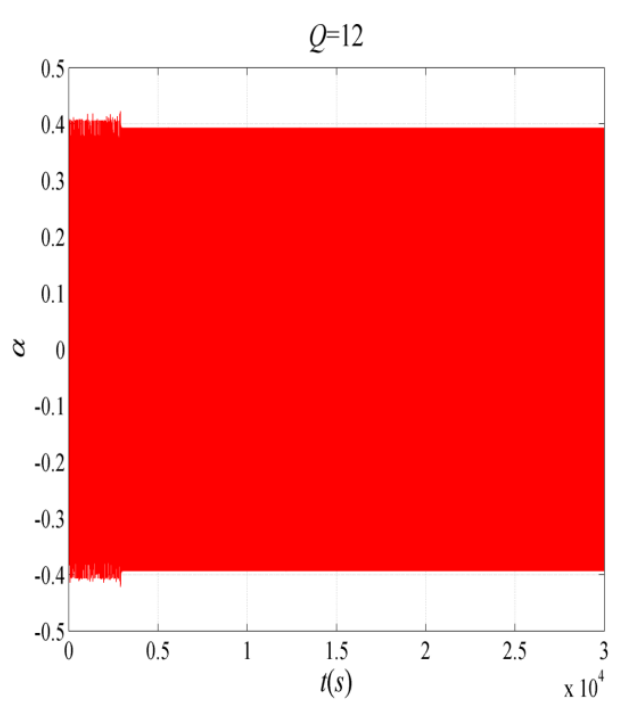

(a)Overall time history

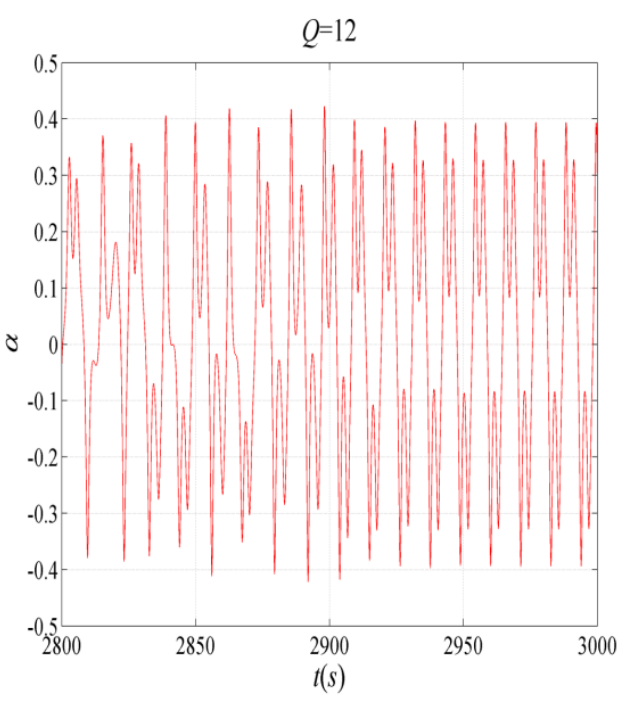

(b)Zoom in parts

Fig.15 The time histories of the airfoil system at $Q=12$

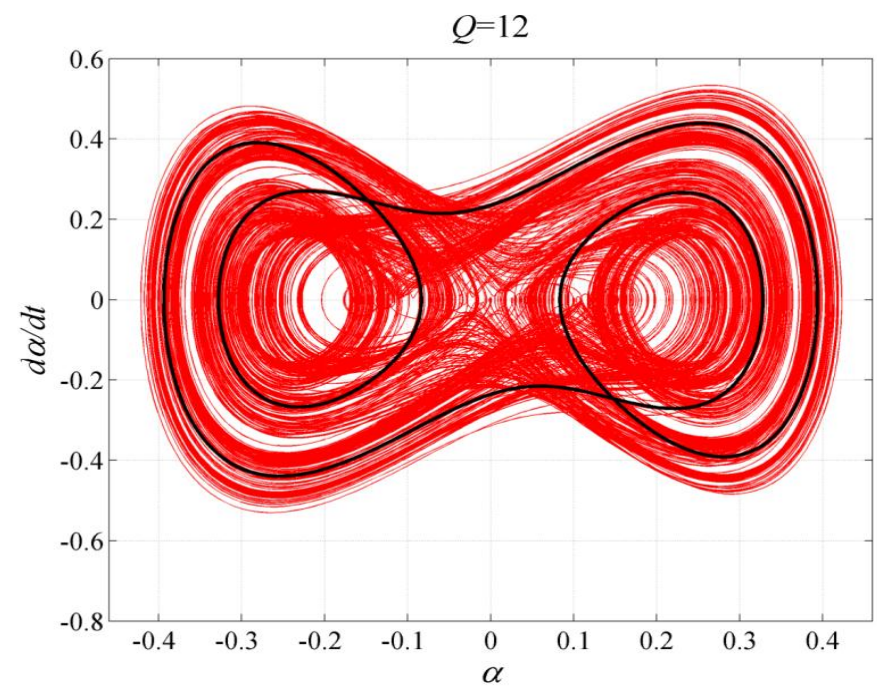

Fig.16 The portraits of the airfoil system at $Q=12$

In the following, the sensitivity of $\frac{d[e(t)]^{1 / 8}}{d Q}$ with $e(t)=\frac{1}{t} \int_{0}^{t}\left[\left(\alpha\left(t^{\prime}\right)\right)^{8}\right] d t^{\prime}$ is analyzed. In order to evaluate $\frac{d[e(t)]^{1 / 8}}{d Q}=\frac{-[e(t)]^{7 / 8}}{8} \frac{d[e(t)]}{d Q}$, the direct differentiation method is used to calculate $\frac{d[e(t)]}{d \beta}$.

By using the direct differentiation method, sensitivity results for the solutions in Figs.14 and 15 are plotted and shown in Fig.17 for $Q=11$ and Fig.18 for $Q=12$. In Figs. 17 and 18, Fig.(b) gives the enlargements of the preceding Fig.(a). As shown in these Figures, sharp 
sensitivity fluctuation at some local regions is detected. In these local regions, the sensitivity of the system may change acutely, which will produce large variance.

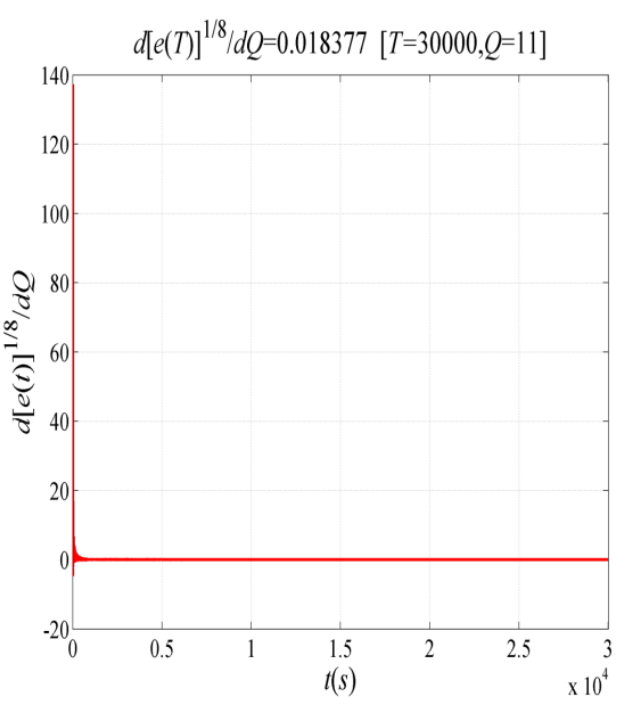

(a)Overall history

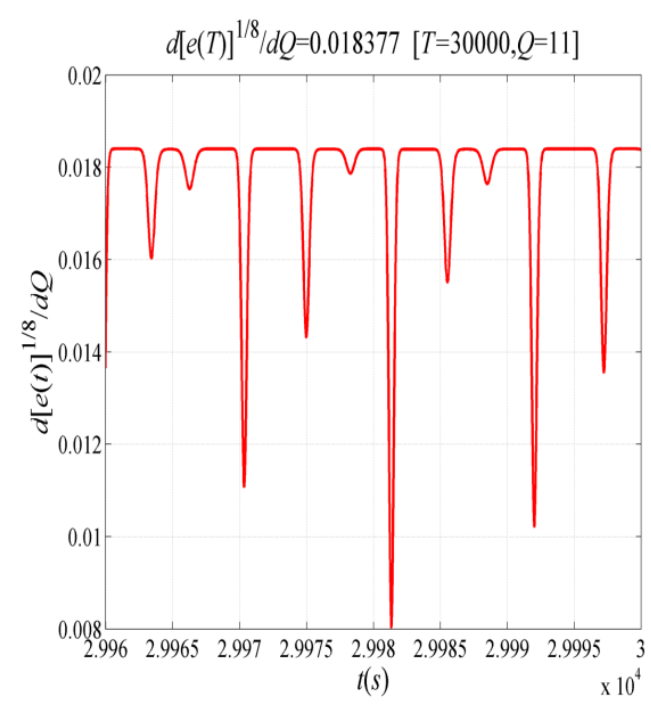

(b) Zoom in parts

Fig.17 The sensitivity results for $Q=11$ obtained by the direct differentiation method

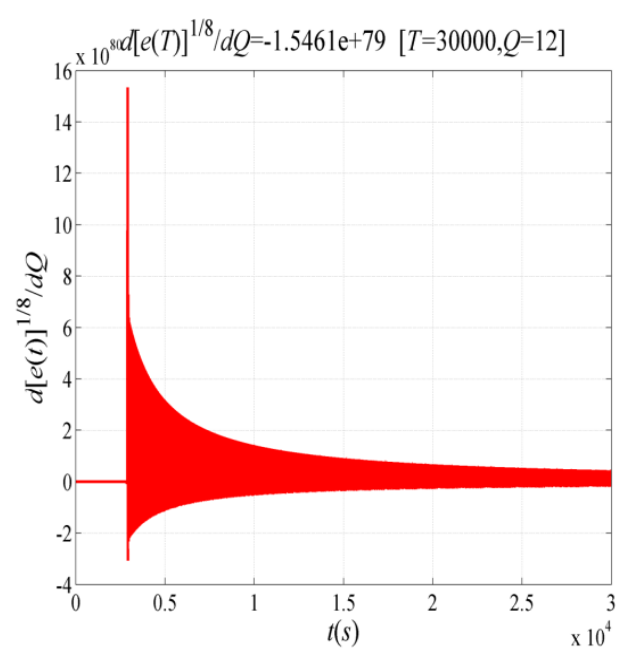

(a)Overall history

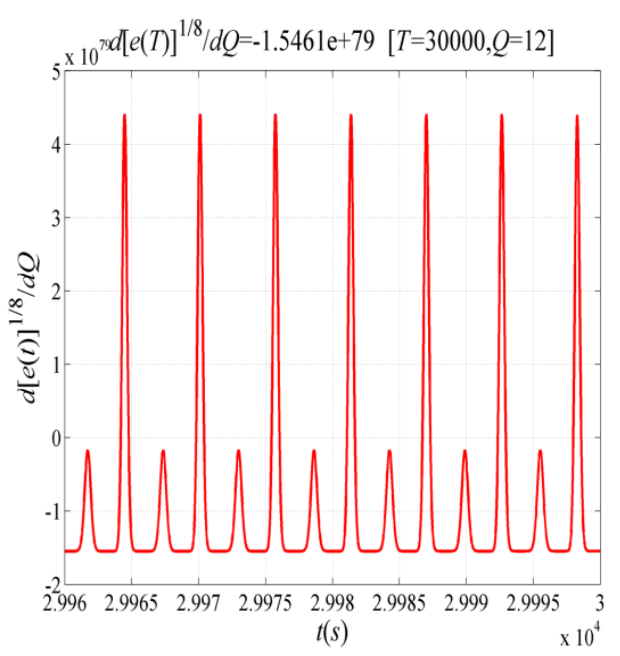

(b) Zoom in parts

Fig.18 The sensitivity results for $Q=12$ obtained by the direct differentiation method

The direct differentiation method produces sensitivity value of 0.18377 for $Q=11$. However, the sensitivity value for $Q=12$ is too large to be usefull. Ill conditions of the sensitivity equations at $Q=12$ may cause this situation. The direct differentiation method thus fails to predict the sensitivity results. To perform the sensitivity analysis over the whole parameter range considered, the direct differentiation method is not suitable for this example.

In the following, the improved LSS method is used to analyze the sensitivity coefficients over the parameter range $Q \in[8,16]$ with step size of 0.2 . For each value of $Q$, the time domain integration solution $\boldsymbol{U}(t)$ for $t \in\left[t_{0}, t_{0}+T\right]$ with fixed step-size 0.01 is first calculated and the initial conditions to obtain the time integration solutions are supplied by the trial numerical simulation with integration time of length 300 .

The sensitivity coefficients resulted from the improved sensitivity analysis method are illustrated in Fig.19 where 20 samples for each $Q$ with $T=300$ are employed to test the robust 
performance of the developed method. In Fig.19, a significant amount of variability of the sample sensitivity distributions is observed at the parameter range between 11 and 12 .

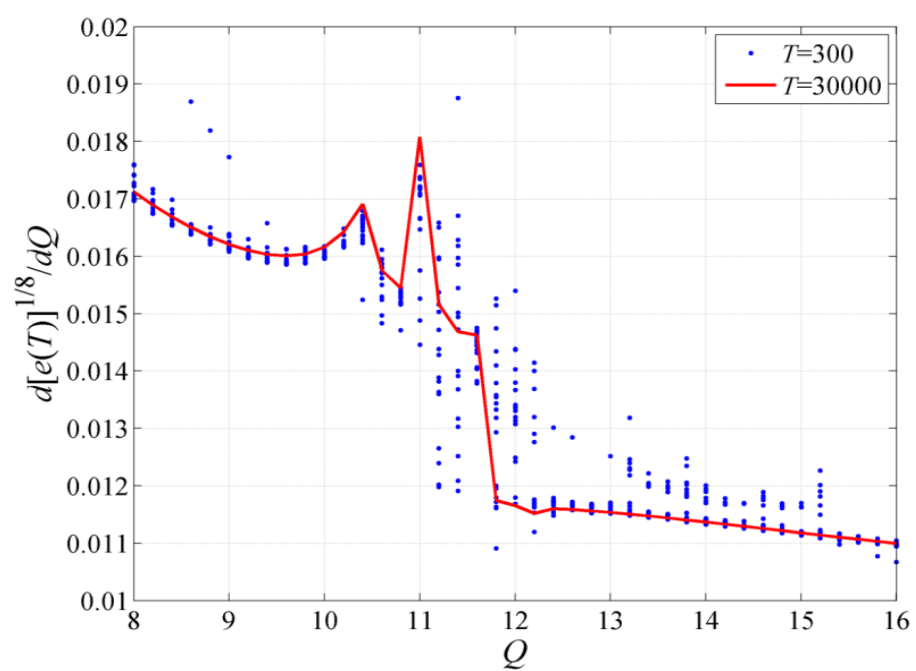

Fig.19 The sensitivity results obtained from the improved least squares shadowing method. The blue dots represent the sensitivity coefficients corresponding to $T=300$. The red line denote the sensitivity coefficients for $T=30000$.

For the red sensitivity curve obtained with $T=30000$ in Fig.19, the sensitivity $\frac{d[e(t)]^{1 / 8}}{d Q}$ decreases with an increase in the flow velocity $Q$ between 8 and 9.6. The sensitivity magnitude decreases to a local minimum value at $Q=9.6$ and then begins to increase. As the flow velocity further increases, the sensitivity value changes significantly within $Q \in$ [10.4,11.8] and sharp peak of the sensitivity coefficient at $Q=11$ is observed. When the flow velocity $Q$ varies from 11.8 to 16 , the sensitivity decreases slowly.

Finally, in comparison with the results of Wang [21], the results in Fig.19 are consistent with the published results given in Fig.8(d) of [21]. The good agreement indicates the validity of the proposed approach.

\section{Conclusions}

An efficient sensitivity analysis scheme is introduced to solve the sensitivity problem of nonlinear systems. The principle is based on the differentiation of time averaged integration term with respect to the time variable, shifting the nonlinear time averaged integration term to a time-independent differential equation. Eventually, the original ordinary differential equation is transformed into an augmented differential system for an easy application of the sensitivity analysis method.

By incorporating the finite difference and trapezoidal discretization scheme, a set of linear equations determined by the first optimal conditions of the improved least squares formulation is obtained and the simple algebraic expression for the response function is derived. With making use of the LU factorization technique, the recursive iterate formula is 
given to compute the final state value of the Lagrange multipliers. The resulted formula used for sensitivity analysis has low computational cost and memory requirements due to the direct dependence of the response function with respect to the final state value of the Lagrange multipliers. Consequently, the sensitivity coefficients are significantly less expensive to evaluate.

The effectiveness of the proposed approach has been demonstrated using three different numerical examples. It was shown that the direct differentiation sensitivity analysis method produces exact sensitivity coefficients for some examples and can capture the fluctuating phenomenon of local sensitivity coefficients. The study results provide valuable insight for engineering practice. Moreover, the numerical results of the improved LSS method coincide perfectly with those found in the literature and the proposed methods are therefore validated.

\section{Acknowledgements}

The financial sponsorships from the National Science Foundation of China(Contract no. 11502261) and the Aeronautical Science Foundation of China(Grant no. 2015ZB04002) are gratefully acknowledged. The author highly acknowledges the proposals provided by the anonymous reviewers who helped to improve the quality of the paper.

\section{References}

[1] T. Lassila, et al, Model order reduction in fluid dynamics: challenges and perspectives, Springer, Switzerland, 9(2014) 235-273.

[2] H. Badreddine, S. Vandewalle, J. Meyers, Sequential Quadratic Programming (SQP) for optimal control in direct numerical simulation of turbulent flow, J. Comput. Phys. 256 (2014) 1-16.

[3] X. Zhang, M.D. Pandey, An effective approximation for variance-based global sensitivity analysis. Rel. Eng. Sys. Safety 121 (2014) 164-174.

[4] H.T. Liao, Uncertainty quantification and bifurcation analysis of an airfoil with multiple nonlinearities, Math. Prob. Eng. 2013, Article ID 570947.

[5] S. Dou, et al, Structural optimization for nonlinear dynamic response, Phil. Trans. R. Soc. A 373 (2015) 20140408.

[6] P. Luchini, A. Bottaro, Adjoint equations in stability analysis, Ann. Rev. fluid mech. 46(1) (2014) 493.

[7] D.G. Cacuci, Sensitivity and uncertainty analysis, volume I: theory, CRC Press, Boca Raton, 2003.

[8] D.G. Cacuci, M. Ionesku-Bujor, I.M. Navon, Sensitivity and uncertainty analysis, volume II: applications to large-scale systems, CRC Press, Boca Raton, 2005.

[9] E. Rosenwasser, R. Yusupov, Sensitivity of automatic control systems, CRC Press, Boca Raton, 2000.

[10] K.A. Wilkins, B. Tidor, J. White, P. Barton, Sensitivity analysis for oscillating dynamical systems, SIAM J. Sci. Comput. 31 (4) (2009) 2706-2732.

[11] H.T. Liao, Optimization analysis of Duffing oscillator with fractional derivatives, Nonlinear Dyn. 79(2) (2015) 1311-1328.

[12] H.T. Liao, W. Sun, A new method for predicting the maximum vibration amplitude of periodic solution of non-linear system, Nonlinear Dyn.71 (3) (2013)569-582. 
[13] H.T. Liao, Nonlinear Dynamics of Duffing Oscillator with Time Delayed Term, CMES: Comp. Model. Eng. Sci. 103(3) (2014) 155-187.

[14] H.T. Liao, Stability Analysis of Duffing Oscillator with Time Delayed and/or Fractional Derivatives, Mech. Bas. Des. Struct. Mach. In press (2015).

[15] H.T. Liao, Piecewise constrained optimization harmonic balance method for predicting the limit cycle oscillations of an airfoil with various nonlinear structures, J. Fluids Struct. 55 (2015) 324-346.

[16] E.N. Lorenz, Deterministic nonperiodic flow, J. Atmos. Sci. 20 (2) (1963) 130-141.

[17] S.A. Soldatenko, P. Steinle, C. Tingwell, D. Chichkine, Some aspects of sensitivity analysis in variational data assimilation for coupled dynamical systems, Adv. Meteo. 2015 (2015) 1-23.

[18] S.A. Soldatenko, R.M. Yusupov, Sensitivity analysis in optimal control of the Earth's climate system, Rec. Adv. Env. Ear. Sci. Econo.2015 (2015) 1-7.

[19] D. Lea, M. Allen, T. Haine, Sensitivity analysis of the climate of a chaotic system,Tellus 52A(2000)523-532.

[20] Q. Wang, Forward and adjoint sensitivity computation for chaotic dynamical systems, J. Comput. Phys. 235(15)(2013)1-15.

[21] Q. Wang, R. Hu, P. Blonigan, Least squares shadowing sensitivity analysis of chaotic limit cycle oscillations, J. Comput. Phys. 267 (2014) 210-224.

[22] K.J. Palmer, Shadowing in dynamical systems: theory and applications, Kluwer, Dordrecht, 2000.

[23] S.Y. Pilyugin, Shadowing in dynamical systems, lecture notes in mathematics, Springer-Verlag, Berlin, 1999.

[24] Q. Wang, Convergence of the least squares shadowing method for computing derivative of ergodic averages, SIAM J. Numer.Anal.52 (1) (2014)156-170.

[25] M. Chater et al, Least Squares Shadowing method for sensitivity analysis of differential equations, arXiv preprint arXiv:1509.02882 (2015).

[26] Q. Wang, P. Blonigan, Multigrid-in-time for sensitivity analysis of chaotic dynamical systems, Numer. Lin. Alg. Appl. (2014).

[27] P. Blonigan, S. Gomez, Q. Wang, Least squares shadowing for sensitivity analysis of turbulent fluid flows, arXiv preprint arXiv:1401.4163 (2014). 